gr-qc/0309117

\title{
Generalized Quantum Theory of Recollapsing Homogeneous Cosmologies
}

\author{
David Craig* \\ Department of Physics, Hamilton College, \\ Clinton, NY 13323 \\ James B. Hartle \\ Department of Physics, \\ University of California \\ Santa Barbara, CA 93106-9530
}

(Dated: October 6, 2018)

\begin{abstract}
A sum-over-histories generalized quantum theory is developed for homogeneous minisuperspace type A Bianchi cosmological models, focussing on the particular example of the classically recollapsing Bianchi IX universe. The decoherence functional for such universes is exhibited. We show how the probabilities of decoherent sets of alternative, coarse-grained histories of these model universes can be calculated. We consider in particular the probabilities for classical evolution defined by a suitable coarse-graining. For a restricted class of initial conditions and coarse grainings we exhibit the approximate decoherence of alternative histories in which the universe behaves classically and those in which it does not. For these situations we show that the probability is near unity for the universe to recontract classically if it expands classically. We also determine the relative probabilities of quasi-classical trajectories for initial states of WKB form, recovering for such states a precise form of the familiar heuristic " $J \cdot d \Sigma$ " rule of quantum cosmology, as well as a generalization of this rule to generic initial states.
\end{abstract}

PACS numbers: 03.65.Ca,03.65.Yz,04.60.Gw,04.60.Kz,04.60.-m,98.80.Qc

\footnotetext{
*E-mail: dcraig@hamilton.edu

†E-mail: hartle@physics.ucsb.edu
} 


\section{INTRODUCTION}

Hamiltonian quantum mechanics with its requisite preferred time(s) may need to be generalized to provide a quantum framework for cosmology where spacetime geometry fluctuates quantum mechanically and does not specify a fixed notion of time. One approach to such a generalization is the sum-over-histories generalized quantum theory of spacetime geometry, described most completely in [1] where references to the earlier literature may be found. This is a formulation of quantum theory in fully four-dimensional spacetime form. The essential elements of this sum-over histories formulation are:

1. Fine-Grained Histories These are the elements of the set of four-dimensional histories of spacetime metric and matter field configurations. They are the most refined descriptions of the universe it is possible to give.

2. Coarse-Grained Histories Partitions of the fine-grained histories into four-dimensional diffeomorphism invariant classes are called coarse-grained histories. Such sets of coarse-grained histories are the most general notion of alternative describable in spacetime terms for which quantum theory predicts probabilities.

3. Decoherence Functional This is a measure of the quantum mechanical interference between members of a set of alternative coarse-grained histories. It is constructed according to sum-over-histories principles and incorporates a theory of the universe's initial and final condition. The decoherence functional is a natural generalization to closed quantum systems of the algebraic notion of quantum state $[2,3]$. Sets of histories with negligible interference between all pairs of members, as measured by the decoherence functional, are said to decohere, or to be consistent. It is logically consistent to assign probabilities in an exhaustive set of alternative histories when, and only when, that set is decoherent. It is the criterion of decoherence, rather than any notion of "measurement", which determines the consistency of the quantitative predictions of the theory.

This paper applies sum-over-histories generalized quantum theory to a class of homogeneous minisuperspace cosmological models. ${ }^{1}$ Other formulations of the quantum mechanics of homogeneous cosmological models have been proposed by Ashtekar, Tate, and Ugla [8], by Wald [9] (see further [10]), and by Marolf [11]. An implementation of generalized quantum theory for these minisuperspace models is therefore useful for comparison with these other approaches. That is especially the case since the formulation of Wald makes the surprising prediction that a quantum universe whose expansion is accurately predicted by classical Einstein dynamics cannot have a nearly classical recontracting phase. (Instead, time appears to "freeze" as the universe approaches its classical maximum size.) In this paper we will show that, in suitable circumstances, sum-over-histories generalized quantum theory predicts that a universe may remain classical in both its expanding and recontracting phases.

Section II revisits the classical homogeneous cosmological models as an introduction to their later quantization. Section III sets out the sum-over-histories generalized quantum mechanics for the classical models of Section II for the especially simple case of a closed Bianchi

\footnotetext{
${ }^{1}$ There is a substantial literature on these models. They were introduced into quantum cosmology by Misner [4]. See MacCallum [5], Halliwell [6], and Wainwright and Ellis [7] for partial guides to the literature.
} 
IX cosmology with a single homogeneous scalar field and vanishing cosmological constant. (Current observations suggest that $\Lambda$ is small but not zero. Assuming $\Lambda$ vanishes, while evidently not realistic, simplifies some elements of the analysis by allowing quantum evolution to be restricted to universes which always recontract. While the quantization framework described in Section III is in principle applicable to all type A Bianchi cosmologies, the case where the universe is allowed to expand forever - either classically or quantum mechanically - will not be considered here.) Section IV explicitly constructs the decoherence functional for these models for a specific class of boundary conditions: a "pure" initial state and "indifferent" final conditions. In Section $\mathrm{V}$ we examine its semiclassical predictions for initial conditions that correspond to a single classical trajectory and show how classical evolution can be an approximation to quantum mechanical evolution in a universe with expanding and contracting phases, as well as study more general choices of initial state.

Generalized quantum theory $[12,13]$ is a broad framework for describing and comparing different formulations of quantum mechanics. A reduction to essentials of the general principles of the quantum mechanics of closed systems [14-16], the framework provides a natural language with which to frame questions in cosmology concerning whether probabilities are consistently assigned by quantum theory to a set of alternative histories of the universe. The specific sum-over-histories implementation of its principles sketched above is but one of several approaches to a conceptually coherent and manageable quantum theory of spacetime; for lucid reviews of some of them and the difficulties they encounter see [17].

\section{HOMOGENEOUS COSMOLOGICAL MODELS}

In this section we review the essential features of homogeneous cosmological models necessary for the subsequent discussion of their quantization. ${ }^{2}$ A spatially homogeneous cosmological geometry is a spacetime possessing a group of isometries whose orbits are a family of spacelike surfaces that foliate the spacetime [20]. Using a coordinate $t$ that labels these spacelike surfaces, the metric of a spatially homogeneous spacetime may be put in the standard form $[20,21]$

$$
d s^{2}=-L^{2}(t) d t^{2}+e^{2 \alpha(t)}\left(e^{2 \beta(t)}\right)_{i j} \sigma^{i} \sigma^{j} .
$$

Here the $\sigma^{i}$ are $t$-independent spatial one-forms preserved by the isometries whose dual vector fields $\sigma_{i}$ obey

$$
\left[\sigma_{i}, \sigma_{j}\right]=c_{i j}^{k} \sigma_{k},
$$

where the $c_{i j}^{k}$ are the components of the structure tensor of the Lie algebra of the isometry group in the $\sigma$ basis. ${ }^{3}$ The quantities $L(t)$ and $\alpha(t)$ are functions of $t$ alone; $\beta(t)$ is a $3 \times 3$ traceless symmetric matrix that measures the deviations from isotropy. The coordinate volume element of the spatial slice scales like $\sqrt{h}=\exp (3 \alpha(t))$ and the same is true for the overall volume $(4 \pi)^{2} \exp (3 \alpha(t))$ if the spatial slices are closed.

The possible spatial isometry groups may be classified by their Lie algebras (e.g. as in [22]) and are usually referred to in the numbering scheme introduced by Bianchi [23] as

\footnotetext{
${ }^{2}$ A classical general reference is [18]. Extended discussions can be found in [5, 19] and in Chap. 7 of [20].

${ }^{3}$ See Wald [20, section 7.2] for example. To avoid possible confusion, note that while Wald's $\sigma$ 's coincide with the $\omega$ 's of MacCallum [5], MacCallum's structure tensor $c$ is defined with the sign opposite to that of Wald.
} 
"Bianchi I" through "Bianchi IX" models. The (simply-connected covering) group manifold with its natural metric is the manifold for spatial geometry. In the Bianchi I models, for example, the group is generated by the translations of three-dimensional flat space and the manifold is $\mathbf{R}^{3}$. In the Bianchi IX models the group is $S U(2), c_{i j}^{k}=\varepsilon_{i j k}$ (in appropriate coordinates), and the manifold is the three-sphere $S^{3}$. The classical Friedmann-RobertsonWalker (FRW) models are the most familiar examples of Bianchi universes: the open FRW universe is of Bianchi type I, the flat universe type $\mathrm{V}$, and the closed FRW universe is of type IX.

The "type A" Bianchi models are those for which $c_{i j}^{i}=0$; the rest are called "type B". We restrict attention to type $\mathrm{A}$ models because the action principle for the type B Bianchi models deduced from that for general relativity by substitution of the homogeneity ansatz (2.1) does not lead to the correct equations of motion [24]; in these cases the homogeneity of the spatial metric obstructs the elimination of boundary terms proportional to the trace of the structure tensor. The Bianchi types I, II, $\mathrm{VI}_{0}, \mathrm{VII}_{0}, \mathrm{VIII}$, and IX are all type A.

A variety of matter contents are consistent with homogeneity. As an illustrative example we shall restrict attention to a single, minimally coupled, homogeneous scalar field $\phi(t)$ together with a positive cosmological constant $\Lambda$. For the action of the scalar field we take

$$
S_{M}[g, \phi]=-\frac{1}{2} \int d^{4} x \sqrt{-g}\left[(\nabla \phi)^{2}+V_{\phi}(\phi)\right]
$$

for some potential $V_{\phi}(\phi)$. [We follow, as far as possible, the conventions of [20] with respect to signature $(-,+,+,+)$, definitions of the curvature tensors, the extrinsic curvature of a hypersurface, etc.. We employ units where $\hbar=c=G=1$.]

A canonical action for the type A Bianchi cosmologies with scalar matter may be arrived at in the following manner. First note that for all the type A models with "diagonal" matter $\left(T_{0 i}=0\right)$, as in our example, it is possible classically to choose the one-forms $\sigma^{i}$ in (2.1) so that the matrix $\beta_{i j}$ is diagonal, and the $c_{i j}^{k}$ take their canonical values $[18,19]$; the Einstein equations guarantee that $\beta$ remains diagonal as time passes. It is then traditional to parametrize $\beta$ as $\beta=\operatorname{diag}\left(\beta_{+}+\sqrt{3} \beta_{-}, \beta_{+}-\sqrt{3} \beta_{-},-2 \beta_{+}\right)$.

Assuming that the metric remains diagonal quantum mechanically is equivalent to solving the classical momentum constraints before quantization [10] ( $c f$. [20, equation E.2.34]); the only remaining gauge freedom then lies in the time-reparametrizations of the surfaces of homogeneity. We shall assume that the metric remains diagonal in the sequel. However, the quantum theory thus obtained is not obviously equivalent to a theory in which the momentum constraint is instead imposed as an operator condition. ${ }^{4}$

Inserting a diagonal homogeneous metric (2.1) into the action $S=(1 / 16 \pi) \int(R-2 \Lambda)+S_{M}$ and doing the spatial integration over a standard coordinate volume of $(4 \pi)^{2}$ (the coordinate volume of Bianchi IX's $S U(2)$ closed spatial manifold; $c f$. [25, Box 30.1]) yields the reduced minisuperspace action

$$
S=\int d t\left[p_{\alpha} \dot{\alpha}+p_{+} \dot{\beta}_{+}+p_{-} \dot{\beta}_{-}+p_{\phi} \dot{\phi}-\left(\frac{L e^{-3 \alpha}}{24 \pi}\right) H\right]
$$

\footnotetext{
4 The issue is that while the momentum constraint implies that the spatial metric may always be diagonalized at any one moment of time, the classical equations of motion are required to show that it remains so thereafter. These issues are discussed with characteristic lucidity in section IV of [10].
} 
where $H$ is the super-Hamiltonian

$$
H=-p_{\alpha}^{2}+p_{+}^{2}+p_{-}^{2}+p_{\phi}^{2}+e^{4 \alpha} V_{\beta}\left(\beta_{+}, \beta_{-}\right)+e^{6 \alpha} V_{\phi}(\phi)+e^{6 \alpha} \Lambda
$$

after rescaling $\phi, V_{\phi}$, and $\Lambda$ by positive constants $\left(\sqrt{4 \pi / 3}, 192 \pi^{3}\right.$, and $48 \pi^{2}$, respectively). The potential $V_{\beta}$ is given in general by

$$
V_{\beta}\left(\beta_{+}, \beta_{-}\right)=-24 \pi^{2} e^{2 \alpha(3)} R\left(\alpha, \beta_{+}, \beta_{-}\right),
$$

where ${ }^{(3)} R$ is the scalar curvature of the homogeneity hypersurfaces. Various explicit expressions for $V_{\beta}$ may be read off from corresponding expressions in [10, 18, 19, 26]. For the characteristic Bianchi IX ("mixmaster" [27]) universe that will be the subject of Sections IV and $\mathrm{V}$ it is [25, Box 30.1, for example]

$$
V_{\beta}=12 \pi^{2} \operatorname{tr}\left[e^{4 \beta}-2 e^{-2 \beta}\right] .
$$

(For ease of comparison with the literature, note that it is traditional to discuss $V_{\beta}$ in terms of the anisotropy potential $V_{a}=\left(1 /(6 \pi)^{2}\right) V_{\beta}+1=\frac{1}{3} \operatorname{tr}\left[1-2 e^{-2 \beta}+e^{4 \beta}\right]$, because $V_{a}$ is positive definite with a global minimum of 0 at $\beta=0$, and is triangularly symmetric about $\beta=0$.) The homogeneous isotropic FRW universe may be recovered by setting $\beta=0$ in the equations for Bianchi IX. For them ${ }^{(3)} R=(3 / 2) e^{-2 \alpha}$ and $V_{\beta}=-(6 \pi)^{2}$. (Note the usual FRW scale factor $a=2 e^{\alpha}$ after changing from the Euler-angle $\sigma^{i}$ basis to the standard polar coordinates on $S^{3}$.)

The configuration space for these minisuperspace models is spanned by the variables $\left(\alpha, \beta_{+}, \beta_{-}, \phi\right)$. That is, it is the superspace of spatial geometries plus the space of scalar field values. The action (2.4) may be expressed in a more compact form by introducing the notation $q^{A}, A=0,1,2,3$ for the four variables $\left(\alpha, \beta_{+}, \beta_{-}, \phi\right)$, the flat Lorentzian DeWitt metric $G_{A B}=\operatorname{diag}(-1,1,1,1)$, and a rescaled lapse function $N(t)=e^{-3 \alpha(t)} L(t) / 24 \pi$. Then

$$
S\left[p_{A}, q^{A}, N\right]=\int_{0}^{1} d t\left[p_{A} \dot{q}^{A}-N H\right]
$$

where

$$
\begin{aligned}
H & =G^{A B} p_{A} p_{B}+e^{4 \alpha} V_{\beta}\left(\beta_{+}, \beta_{-}\right)+e^{6 \alpha}\left[V_{\phi}(\phi)+\Lambda\right] \\
& =G^{A B} p_{A} p_{B}+V\left(\alpha, \beta_{+}, \beta_{-}, \phi, \Lambda\right),
\end{aligned}
$$

and we have taken advantage of the arbitrariness in $t$ as a coordinate label to assign the values 0 and 1 to the ends of the range of integration, a choice we shall make wherever convenient. In this form the analogy with a relativistic particle moving in a potential is clear $[1,28]$ and we shall exploit this in what follows.

The form of the metric (2.1) is left unchanged by reparametrizations of the time $t \rightarrow f(t)$. As already noted, this invariance is the remnant of the four-dimensional diffeomorphism invariance of the full theory of general relativity once the diagonal form (2.1) has been fixed. Correspondingly the action (2.8) is invariant under reparametrization transformations of the form

$$
\begin{aligned}
q^{A}(t) & \rightarrow \tilde{q}^{A}(t)=q^{A}(f(t)) \\
p_{A}(t) & \rightarrow \tilde{p}_{A}(t)=p_{A}(f(t)) \\
N(t) & \rightarrow \tilde{N}(t)=N(f(t)) \dot{f}(t)
\end{aligned}
$$


so long as $f(0)=0$ and $f(1)=1$.

Variation of (2.8) or (2.4) with respect to the multiplier $N$ gives the constraint

$$
H\left(p_{A}, q^{A}\right)=0
$$

between coordinates and their conjugate momenta. Variation with respect to the $p_{A}$ and $q^{A}$ give Einstein's equations of motion. The character of the solutions to these equations - the possible classical histories - depends on the isometry group and the value of the cosmological constant $\Lambda$. For example, in the case of $\Lambda=0$ Bianchi I models we expect cosmologies to expand forever from an initial singularity. More precisely, we expect the extrinsic curvature $K=(3 / L) d \alpha / d t$ (trace of the extrinsic curvature tensor) of the $t=$ constant surfaces of homogeneity to remain positive to the future of an initially singular surface. Bianchi I classical solutions with $\Lambda>0$ also expand forever, while models with $\Lambda<0$ always recollapse. Bianchi IX models have closed spatial sections with three-sphere topology. The $\Lambda=0$ Bianchi IX universes are just the anisotropic generalizations of the closed FRW universe. More precisely, Lin and Wald [29] have shown that when the dominant energy condition is satisfied and the trace of the spatial projection of the stress-energy tensor (i.e. the sum of the principal pressures) is positive - thus excluding a cosmological constant - there are no classical solutions which expand forever from an initial singularity in the sense that the extrinsic curvature of constant $t$ surfaces remains positive.

The stress-energy tensor arising from (2.3) satisfies the dominant energy condition so long as $V_{\phi} \geq 0$, though the pressures may be negative if the potential energy in the scalar field exceeds the kinetic energy. (Indeed, it is precisely this feature which allows a scalar field to mimic a cosmological constant in inflationary models.) Thus, scalar fields only satisfy the conditions of the Lin-Wald recollapse theorem at late times for certain choices of scalar potential - a free, massless, minimally coupled scalar field, for example. The general conditions on $V_{\phi}$ for which a recollapse theorem holds are as far as we are aware not currently known.

With a cosmological constant the conditions of the Lin-Wald theorem are not satisified. The example of de Sitter space is enough to show that there will be Bianchi IX solutions with vanishing scalar field which evolve non-singularly from an initial contracting phase $(K<0)$ in the infinite past to an expanding phase $(K>0)$ in the far future. In between the volume of the universe reaches a non-zero minimum value. The inclusion of a small amount of homogeneous scalar field would not be expected to disturb this behavior. However, the homogeneous, isotropic Bianchi IX (Friedmann) models show that a positive cosmological constant does not remove the singularity in every case. There are also models in which the stress-energy of the scalar field dominates that of the cosmological constant throughout the model's history. Such models display the qualitative features of the $\Lambda=0$ case - an initial singularity leading to a finite expansion followed by recontraction, for models satisfying the conditions of the Lin-Wald theorem. See [7] for a wide-ranging survey of the dynamics of the various cosmological models. 


\section{GENERALIZED QUANTUM MECHANICS OF $\Lambda=0$ BIANCHI IX UNI- VERSES}

\section{A. Generalized Quantum Theory}

In this section we describe a generalized quantum theory for Bianchi IX minisuperspace cosmological models with $\Lambda=0$. While the construction is in principle valid even for models which may expand forever, certain technical aspects of the analysis are simplified by restricting attention to quantum histories which always recontract, and the most general case will not be considered here.

We work within the general principles of the quantum mechanics of a closed system [1416]. The closed system is most generally and accurately the universe as a whole. The most general predictions of quantum mechanics are the probabilities of individual members of sets of alternative coarse-grained histories of the closed system. Probabilities are not predicted for every set of alternative histories, but only those for which the quantum mechanical interference between the individual histories in the set is negligible as a consequence of the system's boundary conditions and dynamics. Such sets of histories are said to decohere, or be consistent.

Generalized quantum theory $[1,12,13]$ is a comprehensive framework for implementing the principles of the quantum mechanics of closed systems. As noted in the introduction, the following elements specify a generalized quantum mechanics: (1) The sets of fine-grained histories which are the most refined description of the system possible. (2) The allowed coarse-grainings which generally are partitions of a set of fine-grained histories into an exhaustive set of mutually exclusive classes $\left\{c_{h}\right\}, h=1,2,3 \cdots$ called coarse-grained histories. (3) A decoherence functional, $D\left(h, h^{\prime}\right)$ that measures the interference between pairs of histories in a coarse-grained set. The decoherence functional is a complex-valued functional on pairs of classes that satisfies certain general requirements: It is (i) Hermitian, (ii) normalized, (iii) positive, and (iv) consistent with the principle of superposition in senses made precise in $[1,12,13]$. The decoherence functional incorporates a specification of the boundary conditions for the closed system - typically "initial" and "final" conditions. It is a natural generalization to closed systems of the idea of quantum state, as the term is used in quantum logic and in algebraic quantum mechanics, to measure the quantum interference between histories in addition to their probabilities $[2,3]$.

With these three elements specified, the process of prediction proceeds as follows: A set of alternative coarse-grained histories (approximately, medium) decoheres when $D\left(h, h^{\prime}\right)$ is negligible for all $h \neq h^{\prime}$. The probabilities $p(h)$ of the individual histories in a decoherent set are the diagonal elements of $D$. The rules for decoherence and probabilities are thus summarized by the fundamental formula

$$
D\left(h^{\prime}, h\right) \approx p(h) \delta_{h^{\prime} h}
$$

obeyed by histories in decohering sets.

The four-dimensional diffeomorphism invariance characteristic of a geometric theory of gravity is most easily accomodated by employing a sum-over-histories formulation of quantum mechanics. A sum-over-histories quantum mechanics posits a unique set of fine-grained histories which in the case of gravity are four-dimensional spacetime metrics and matter field configurations. A sum-over-histories generalized quantum mechanics for cosmology was described in [1]. Generalized quantum mechanics for models with a single reparametrization 
invariance was described as well. ${ }^{5}$ We now apply those discussions to homogeneous, minisuperspace cosmological models which, as formulated here, possess a single time reparametrization invariance. To do that we specify explicitly the three elements of their generalized quantum mechanics.

\section{Fine-grained Histories}

We take for the set of fine-grained histories the paths $z^{M}(t)=\left(q^{A}(t), N(t)\right), M=0, \ldots, 4$ in the extended configuration space $\mathcal{C}_{\text {ext }}$ of $q^{A}=\left(\alpha, \beta_{+}, \beta_{-}, \phi\right)$ and multiplier $z^{4}=N$. We are thus considering a sum-over-histories quantum mechanics in which there is a unique finegrained set of histories. We put no restriction of single-valuedness on the paths. Thus, for example, the total spatial volume $(4 \pi)^{2} \exp (3 \alpha(t))$ may increase and decrease over the course of the history and, indeed, go through such cycles an arbitrarily large number of times. In particular, the classical histories of a Bianchi IX universe which expand and recontract are included among the possible quantum mechanical histories.

We put no restriction on the differentiability of the paths, but do require them to be continuous.

The fine-grained histories have ends at which the cosmological boundary conditions analogous to initial and final conditions are imposed, and these ends must be prescribed to complete the specification of the set of fine-grained histories. A natural principle restricting this choice is that the set of fine-grained histories should include all the classical histories. Otherwise there is no hope of recovering Einstein's classical theory as a suitable limit of quantum theory. (This will be the subject of Section V.) The work of Lin and Wald [29] discussed at the end of section II shows that all classical Bianchi IX cosmologies with scalar field and $\Lambda=0$ expand from an initial singularity of vanishing three-volume and eventually recontract to a final singularity of vanishing three-volume, assuming that the potential energy in the scalar field does not dominate the kinetic at late times. We shall confine our attention to potentials for which this is so. Classical histories may therefore be thought of as beginning and ending on a surface $\sigma_{0}$ of large constant negative $\alpha\left(=\alpha_{0}\right)$ in the configuration space of paths. The class of all paths that begin and end on such a surface is therefore the natural, minimal set of fine-grained histories for a generalized quantum theory of Bianchi IX minisuperspace cosmological models with scalar field and $\Lambda=0$. This was the choice advocated by Teitelboim in his theory of quantum cosmological "scattering" between initial and final singularities [30]. It is the choice we shall adopt here. (Restricting the paths in this way corresponds to the imposition of a boundary condition that wave functions vanish

\footnotetext{
${ }^{5}$ The generalized quantum mechanics for cosmology in [1] is but one of many applications of sum-overhistories principles and techniques to quantum gravity. These are too numerous and familiar to be cited here, but two particular applications to quantum cosmological dynamics should be mentioned whose spirit is consonant with that in [1]. C. Teitelboim [30] developed a sum-over-histories " $S$-matrix" theory for calculating transition amplitudes between alternatives defined near cosmological singularities. We exploit the formal techniques developed by Teitelboim for constructing functional integrals over spacetime geometries and in particular his choice for the measure in such integrals. The way probabilities are related to amplitudes differs and we aim, in a generalized quantum mechanical setting, at probabilities for much more general and accessible alternatives than those that can be defined near singularities. R. Sorkin's [31] treatment of general alternatives is concordant with that of the present work.
} 
as $\alpha \rightarrow \infty$.) Finally, we add the restriction that all paths possess $\dot{\alpha}(0)>0$ and $\dot{\alpha}(1)<0$ on $\sigma_{0}$, i.e. "expanding" initial and "contracting" final conditions.

\section{Coarse-grained Histories}

Coarse-grained histories correspond to the physical questions that may be asked of a system. We therefore allow as coarse-grained sets of alternative histories any partition of the fine-grained histories into reparametrization invariant classes $\left\{c_{h}\right\}, h=1,2, \cdots$, because reparametrization invariance is what remains in minisuperspace of the diffeomorphism invariance of general relativity. The classes generally may be thought of as partitions by values of reparametrization invariant functionals $F\left[q^{A}, N\right]$ of the paths in $\mathcal{C}_{\text {ext }}$. Explicitly, for an exclusive set of ranges $\left\{\Delta_{h}\right\}, h=1,2, \cdots$ of the real line, and a single functional $F$ we define

$$
c_{h}=\left\{\left(q^{A}(t), N(t)\right) \mid F\left[q^{A}, N\right] \in \Delta_{h}\right\} .
$$

Any partition may be thought of as of this form because we may always consider the functional which has the value $h$ for paths in $c_{h}$ and a set of ranges that bracket the integers.

Simple examples of interesting partitions into diffeomorphism invariant classes - here reduced to reparameterization invariant classes - are readily given:

- One could partition the histories by ranges of values of the volume of the largest volume three surface of homogeneity. The resulting probabilities are for the values of the volume of maximum expansion of the universe reached in the course of its expansion and contraction.

- One could partition the histories of these homogeneous space times into the classes which have a surface of homogeneity with a volume less than $\epsilon$ and the class of those with no such surface. The value of the probability that there is a surface with volume less than $\epsilon$ considered as $\epsilon \rightarrow 0$ would be one way of assigning a probability to the universe becoming singular. ${ }^{6}$

\footnotetext{
${ }^{6}$ There could be many other meanings assigned to a singular quantum evolution, for example, a finite probability for a curvature invariant to have a divergent value (cf. [32]). The various possible definitions are not necessarily easily related or of equal significance because the paths are non-differentiable. The particular criterion for singular evolution - finite probability for a surface of homogeneity of vanishing volume - that is under discussion for these minisuperspace models would not be a valid criterion for a full theory of quantum cosmology for two reasons: First a general geometry may have no surfaces of homogeneity. Second, spacelike surfaces of nearly vanishing volume may be constructed even in nonsingular spacetimes from segments that are nearly null. The criterion is meaningful in these minisuperspace models only because all fine-grained histories can be foliated by surfaces of homogeneity — by assumption. The important point, however, is that any meaningful criterion for singular quantum evolution that is expressible in spacetime form corresponds to a partition of the fine-grained histories into the class which is singular by that criterion and the class which is not singular. If the alternatives of $\{$ (singular), (nonsingular)\} decohere, then a generalized quantum theory will assign a probability to a quantum universe being singular. Unlike the classical theory, however, a finite probability for singular geometry need not mean a breakdown of predictability.
} 
- One could partition the histories into the class which remain close to a solution of the classical Einstein equation by some standard ( $c f .(5.3))$ and the class which exhibit a significant excursion away from classical behavior. The probability of the first class is the probability that the universe behaves classically according to the given standard. We shall employ such coarse grainings in Section V.

- One could partition the fine-grained histories by ranges of values of the volume and anisotropy $\beta_{ \pm}$they assume a given "proper time" $7 \int L d t$ after the initial condition. The resulting probabilities would be the probabilities for the volumes and anisotropies the universe could have at a given proper time from the initial surface. These are not unlike the probabilities that would be of interest in comparing the predictions of a realistic quantum cosmology with observation.

All of the coarse grainings mentioned above are into manifestly reparametrization invariant classes of fine-grained histories. Most are partitions that are not defined by alternatives that are in any sense alternatives "at one moment of time", and hence are not defined simply by observables on superspace. Rather they are spacetime alternatives referring to properties of histories extended over time. For example, the probabilities for classical behavior refer to whether a suitably coarse-grained history approximately obeys the Einstein equation over a course of time.

Coarse-grained histories correspond to the physical questions that may be asked of a system in the following sense. Ask for the probability that the universe has any reparametrization invariant property expressible in spacetime terms. ${ }^{8}$ To answer this question one considers the partition of the fine-grained histories into the class which have the property and the class which do not. If this set of coarse-grained histories decoheres, then the quantum mechanics predicts the probability that the universe has the property in question. If one cannot tell whether a given fine-grained history has the property or not then either it does not make sense or it is not expressible in terms of metric and/or matter field configurations alone.

While it is easy to exhibit physically interesting sets of alternative reparametrization invariant coarse-grained histories for these homogeneous minisuperspace cosmological models it is much harder to find sets of such histories that decohere. That is because of the small number of degrees of freedom of the model. Coarse-graining is essential for decoherence. Realistic mechanisms of decoherence that are effective in a variety of initial conditions qualitatively involve the dissipation of phases from variables followed by the coarse-graining into variables that are ignored. ${ }^{9}$ However, the present minisuperspace models do not present many degrees of freedom to be ignored!

\footnotetext{
${ }^{7}$ We follow terminology of Teitelboim [30] and Henneaux and Teitelboim [33] in calling the reparametrization invariant quantity $\int L d t$ the "proper time". K. Kuchař [34] has stressed that this may be confusing and suggests the term "separation".

${ }^{8}$ In path integral formulations of this kind, physical quantities that involve derivatives such as the extrinsic curvature $K$ must be expressed in terms of differences of metric variables at different times.

${ }^{9}$ See, e.g. $[35,36]$ for more quantitative discussions from a histories point of view, as well as [37].
} 


\section{The Decoherence Functional}

The decoherence functional is constructed in two steps following the analogy of the relativistic particle discussed in [1]; the final result is expressed in (3.9). It will turn out to be a natural generalization to a reparametrization invariant theory of the canonical decoherence functional of ordinary quantum mechanics.

First we define matrix elements of class operators corresponding to individual coarsegrained histories $h$ by a sum-over-histories in the class. Schematically, we define

$$
\left\langle q^{\prime \prime}\left\|C_{h}\right\| q^{\prime}\right\rangle=\sum_{\text {paths } \in\left[q^{\prime} h q^{\prime \prime}\right]} \exp (i S[\text { path }])
$$

where the sum is over paths in $\mathcal{C}_{\text {ext }}$ that lie in the class $c_{h}$ which begin at $q^{\prime}$ and end at $q^{\prime \prime}$. $S$ is the (Lagrangian) action for paths - a functional of $q(t)$ and $N(t)$. In order to give a definite meaning to the functional integral in (3.3), and, in particular, to fix the "measure" on the space of paths, it is convenient to consider the corresponding integral over paths in phase-space,

$$
\left\langle q^{\prime \prime}\left\|C_{h}\right\| q^{\prime}\right\rangle=\int_{c_{h}} \delta N_{\geq 0} \delta p \delta q_{q^{\prime}}^{q^{\prime \prime}} \Delta_{\mathcal{G}}[q, N] \delta[\mathcal{G}[q, N]] \exp \left(i S\left[p_{A}, q^{A}, N\right]\right)
$$

The ingredients in this expression are as follows: $S$ is the action (2.8). $\mathcal{G}$ is a function such that the condition $\mathcal{G}=0$ fixes a unique representative from each reparametrization invariant equivalence class of fine-grained histories; $\Delta_{\mathcal{G}}$ is the associated Faddeev-Popov determinant. The integral is over all paths in $\mathcal{C}_{\text {ext }}$ that lie in the class $c_{h}$ and pass between the configuration space points $\left(q^{A}\right)^{\prime}$ and $\left(q^{A}\right)^{\prime \prime}$. All possible momentum paths are integrated over. We take the multiplier functional integral to be over positive values $N \geq 0$. Other choices, for example, both positive and negative values, would lead to different generalized quantum theories. (Some of the issues that arise in choosing the allowed range for the lapse are discussed in [38-41] and [1, section VII].) The measure for the $q$ and $p$ integrations is the usual Liouville " $d q d p / 2 \pi$ " measure on phase-space paths. The integration over momenta may be seen as a device to induce the measure on paths in $\mathcal{C}_{\text {ext }}$ from the Liouville measure since the range of integration over the momenta are constrained neither by the class $c_{h}$, the endpoints $\left(q^{\prime}, q^{\prime \prime}\right)$, or the gauge fixing delta-function. The skeletonized path integrals we envision are quite standard [30, 39], and have already been briefly described in [1].

The decoherence functional is constructed from the class operators (3.4) which characterize the physical histories in question, but also incorporates a theory of cosmological boundary conditions imposed at the ends of the histories that are the analogs of initial and final conditions in a quantum system with a fixed notion of time.

An initial or final condition is represented by a set of configuration-space wave functions together with a probability for each wave function. That is the same kind of information needed to specify an initial or final density matrix in ordinary quantum mechanics. The wave functions are required to satisfy an operator implementation of the constraints. Thus, for homogeneous cosmological models an initial condition is specified by a set $\left\{\Psi_{i}\left(q^{A}\right), p_{i}^{\prime}\right\}$, where each $\Psi_{i}\left(q^{A}\right)$ satisfies an operator form of (2.11). We take the obvious operator ordering ${ }^{10}$

\footnotetext{
10 Most generally, the operator ordering in the Wheeler-DeWitt equation obeyed by the boundary wave
} 
and write

$$
H \Psi_{i}(q)=\left[-G^{A B} \frac{\partial^{2}}{\partial q^{A} \partial q^{B}}+V(q)\right] \Psi_{i}(q)=0 .
$$

Similarly a final condition is specified by a set $\left\{\Phi_{j}\left(q^{A}\right), p_{j}^{\prime \prime}\right\}$, where the $\Phi_{j}\left(q^{A}\right)$ also solve the Wheeler-DeWitt equation (3.5). In order to guarantee the independence of the decoherence functional from the choice of initial surface $\sigma_{0}$ - see below (3.8) - and for additional reasons that will be explained in the sequel, we require the initial solutions $\Psi(q)$ of the WheelerDeWitt equation to decay in "spatial" directions in minisuperspace (that is, on surfaces of constant $\alpha$ ) fast enough in $\beta_{ \pm}$and $\phi$ that $V \Psi \approx 0$ as $\alpha \rightarrow-\infty$. (More precisely, we require that $e^{-4 \alpha} V \Psi$ is bounded; see section IV C.) The space of functions on minisuperspace with these properties will be denoted $\mathcal{F}\left(\sigma_{0}\right)$. (3.5) is the Wheeler-DeWitt equation for the "initial" and "final" conditions in this minisuperspace quantum cosmology.

At this stage in the construction of the decoherence functional, the specification of the minisuperspace boundary conditions is otherwise essentially arbitrary. Ultimately, these boundary conditions must be supplied by a detailed physical theory of the cosmological boundary conditions. [6, 42] are reviews of this aspect of quantum cosmology.

Initial and final conditions are adjoined to the class operator matrix elements by means of a linear product $\circ$ on the space of solutions to (3.5). Following [1] we shall take for $\circ$ the Wheeler-DeWitt (Klein-Gordon) product. Specifically, given a surface $\sigma$ in the configuration space that is spacelike in the metric $G^{A B}$ we define

$$
\Phi \circ \Psi=i \int_{\sigma} d \Sigma^{A} \Phi^{*}(q) \stackrel{\leftrightarrow}{\nabla}_{A} \Psi(q) .
$$

The inner product, so defined, is independent of the surface used to define it so long as $\Phi$ and $\Psi$ both satisfy (3.5). In the coordinates $q^{A}=\left(\alpha, \beta_{+}, \beta_{-}, \phi\right)$ in which $G^{A B}=\operatorname{diag}(-1,1,1,1)$ a convenient choice of $\sigma$ is typically a surface of constant $\alpha$, whence

$$
\Phi \circ \Psi=i \int_{\alpha} d^{3} q \Phi^{*}(\alpha, \vec{q}) \frac{\stackrel{\leftrightarrow}{\partial}}{\partial \alpha} \Psi(\alpha, \vec{q})
$$

Having introduced the product $\circ$ we now define

$$
\left\langle\Phi_{i}\left|C_{h}\right| \Psi_{j}\right\rangle=\Phi_{i}\left(q^{\prime \prime}\right) \circ\left\langle q^{\prime \prime}\left\|C_{h}\right\| q^{\prime}\right\rangle \circ \Psi_{j}\left(q^{\prime}\right)
$$

This definition appears at first sight to depend on the choice of surface on which $\circ$ is defined, but in fact it does not, so long as the coarse graining $c_{h}$ does not restrict the paths on those surfaces. This is because the class operators (3.4) for such coarse grainings generally solve (3.5) as well as $\Psi$ and $\Phi$, a fact we demonstrate in the appendix. First, however, we complete the definition of the generalized quantum theory of homogeneous minisuperspace cosmologies.

functions should agree with the operator ordering in the Wheeler-DeWitt equation obeyed by the full propagator, (A.1). This ordering in turn is determined by the precise form of the measure in the functional integral (3.4) defining the class operators. In the present instance, however, we have effectively circumvented this issue at the classical kinematical level by restricting attention to an action of the form (2.9) expressed in coordinates in which the kinetic term is independent of the minisuperspace coordinates; the operator ordering appropriate to this choice is just that of (3.5). 
The decoherence functional $D\left(h^{\prime}, h\right)$ is defined through

$$
D\left(h^{\prime}, h\right)=\mathcal{N} \sum_{i, j} p_{i}^{\prime \prime}\left\langle\Phi_{i}\left|C_{h^{\prime}}\right| \Psi_{j}\right\rangle p_{j}^{\prime}\left\langle\Phi_{i}\left|C_{h}\right| \Psi_{j}\right\rangle^{*}
$$

where $\mathcal{N}$ is a normalizing factor determined so that $\Sigma_{h^{\prime} h} D\left(h^{\prime}, h\right)=1$. Specifically, if $u$ is the class of all fine-grained histories

$$
\mathcal{N}^{-1}=\sum_{i j} p_{i}^{\prime \prime}\left|\left\langle\Phi_{i}\left|C_{u}\right| \Psi_{j}\right\rangle\right|^{2} p_{j}^{\prime} .
$$

The decoherence functional defined by (3.9) is the direct analogue of the "canonical" decoherence functional of ordinary Hamiltonian quantum mechanics with initial and final boundary conditions $[1,3]$, written in functional integral form, with accomodations appropriate to the reparametrization invariance of the present theory. It satisfies the general conditions required of a generalized quantum theory. It is (i) Hermitian, $D\left(h^{\prime}, h\right)=D\left(h, h^{\prime}\right)^{*}$, (ii) normalized, $\Sigma_{h h^{\prime}} D\left(h^{\prime}, h\right)=1$, (iii) positive on the diagonal elements, $D(h, h) \geq 0$, and, (iv) consistent with the principle of superposition in the sense that if $\left\{\bar{c}_{\bar{h}}\right\}$ is a partition of the classes $\left\{c_{h}\right\}$ into coarser classes, then

$$
D\left(\bar{h}^{\prime}, \bar{h}\right)=\sum_{h^{\prime} \in \bar{h}^{\prime}} \sum_{h \in \bar{h}} D\left(h^{\prime}, h\right)
$$

These four conditions are enough to ensure that for sets of histories that decohere according to (3.1), the numbers $p(h)$ defined by (3.1) are probabilities satisfying the most general form of the probability sum rules. By using in (3.1) the specific form (3.9) we can assess the probabilities of alternative, coarse-grained, decohering histories of the model homogeneous cosmologies under discussion.

\section{B. Evaluation of the Class Operators in the Proper Time Gauge}

We apply (3.1) to predictions concerning the semiclassical behaviour of homogeneous minisuperspace cosmologies in the next section. To end this section, we discuss the evaluation of the class operators (3.4) in a particularly convenient gauge - (3.13) - called the "proper time" gauge. For suitable coarse grainings, we also briefly argue that these matrix elements satisfy the constraint (3.5). Because the action (2.8) is essentially that of a relativistic particle in a potential, the treatment closely parallels that of the free relativistic particle that has been given previously in [1, section 7$]$.

The first step in evaluating (3.4) is to choose a "gauge" that fixes the reparametrization symmetry (2.10a-2.10c), the infinitesimal form of which is invariance under the changes (set $f(t)=t+\epsilon / N)$

$$
\begin{aligned}
\delta q^{A} & =\epsilon(t)\left\{q^{A}, H\right\} \\
\delta p_{A} & =\epsilon(t)\left\{p_{A}, H\right\} \\
\delta N & =\dot{\epsilon}(t),
\end{aligned}
$$

where $\{$,$\} is the Poisson bracket, and \epsilon(0)=\epsilon(1)=0$. A convenient "gauge" fixing function is $[38,40]$ (see also $[1$, section 7$]$ )

$$
\mathcal{G}=\dot{N} .
$$


The Fadeev-Popov determinant $\Delta_{\mathcal{G}}=\operatorname{det} \delta \mathcal{G} / \delta \epsilon \sim \operatorname{det} d^{2} / d t^{2}$ is in this case independent of the integration variables. The delta functional in (3.4) then permits only the $N=$ constant paths to contribute to the integral over $N(t)$, leaving

$$
\left\langle q^{\prime \prime}\left\|C_{h}\right\| q^{\prime}\right\rangle=\int_{c_{h}} d N_{\geq 0} \delta p \delta q_{q^{\prime}}^{q^{\prime \prime}} \exp \left(i \int_{0}^{1} d t\left[p_{A} \dot{q}^{A}-N H\right]\right)
$$

after dropping the constant factors that cancel in the decoherence functional (3.9). Changing variables to

$$
d s=N d t,
$$

the Gaussian functional integrals over $p$ may be performed, leaving simply

$$
\left\langle q^{\prime \prime}\left\|C_{h}\right\| q^{\prime}\right\rangle=\int_{0}^{\infty} d N\left\langle q^{\prime \prime} N\left\|C_{h}\right\| q^{\prime} 0\right\rangle
$$

so long as we assume the coarse graining does not restrict the value of $N$; otherwise the range of the $N$ integral must be restricted appropriately as well. Here we have defined

$$
\begin{aligned}
\left\langle q^{\prime \prime} N\left\|C_{h}\right\| q^{\prime} 0\right\rangle & =\int_{c_{h}} \delta q_{q^{\prime}}^{q^{\prime \prime}} \exp \left(i \int_{0}^{1} d t\left[\frac{1}{4 N} G_{A B} \dot{q}^{A} \dot{q}^{B}-N V\right]\right) \\
& =\int_{c_{h}} \delta q_{q^{\prime}}^{q^{\prime \prime}} \exp \left(i \int_{0}^{N} d s\left[\frac{1}{4} G_{A B} \frac{d q^{A}}{d s} \frac{d q^{B}}{d s}-V\right]\right)
\end{aligned}
$$

where the path integral measure $\delta q$ has been renormalized in the usual manner induced by the momentum integrations. (See the appendix for details.) The notation on the left hand side of $(3.17 \mathrm{a})$ is inspired by the observation that the path integral in (3.17b) shares the form of that for the propagator over a time $N$ of a relativistic particle in a potential $V$. (3.16) may be thought of as a "restricted propagator" for the class of paths $c_{h}$, as should be evident from the restricted functional integral (3.4).

In general, the complexity of the minisuperspace potential $V$ precludes much further explicit progress in the non-perturbative evaluation of $\left\langle q^{\prime \prime}\left\|C_{h}\right\| q^{\prime}\right\rangle$. It is still possible, however, to show that the class operators satisfy the constraint (3.5) for coarse grainings which do not restrict the values of the endpoints $q^{\prime}$ and $q^{\prime \prime}$ or the value of $N .{ }^{11}$ This is done in the appendix. (3.8) is thus as claimed independent of the surfaces on which we choose to impose the boundary conditions $\Psi$ and $\Phi$.

\section{DECOHERENCE FUNCTIONAL FOR RECOLLAPSING BIANCHI COS- MOLOGIES}

The general form of the decoherence functional for type A homogeneous cosmologies is given in (3.9). In this section we employ some specific choices of the initial and final conditions approriate to $\Lambda=0$ closed (Bianchi IX) cosmologies to cast the decoherence functional into a simpler and more practically useful form.

11 Another approach is to require that the class operators satisfy the constraints and to modify their definition appropriately [28, 43-45]. We do not expect such modifications to fundamentally affect any of the specific results presented here, and thus defer their consideration. 


\section{A. Initial and Final Conditions}

As already noted, it is to be expected that the initial quantum conditions of the universe are fixed by some theory of cosmological boundary conditions. Knowledge of this theory is not, however, required in the construction of the decoherence functional for cosmology. We shall, therefore, illustrate the process of prediction based on the decoherence functional (3.9) with some simple choices of initial state. The practical significance of such predictions depends entirely on whether the chosen initial states are representative of the boundary conditions on the actual physical universe.

Most of the extant theories of the initial state of the universe [6, 42] produce a boundary state consisting in a single initial wave function. We will therefore in our examples concentrate entirely on the case of a pure initial state $\Psi$.

Recall from Section III A 1 that the Bianchi IX cosmological histories have "ends" at which we impose boundary conditions $\left\{\Psi_{i}, p_{i}^{\prime}\right\}$ and $\left\{\Phi_{i}, p_{i}^{\prime \prime}\right\}$. In order to correspond to a conventional notion of cosmological boundary conditions for closed, $\Lambda=0$ universes, we impose the boundary conditions on a suitable surface $\sigma_{0}$ of large, negative $\alpha\left(=\alpha_{0}\right)$, i.e. when the universe is very small. Because the wave functions $\Psi_{i}$ and $\Phi_{i}$ satisfy the constraint (3.5), it does not matter on which surface they are imposed so long as the coarse grainings under consideration do not involve regions of minisuperspace intersecting those surfaces, as noted above. In this sense, then, the cosmological histories "begin" and "end" at small spatial volume, just as the classical histories do. (See Figure 1.)

Maintaining a close correspondence with ordinary quantum mechanics, we shall choose final boundary conditions which are "indifferent" to the paths, in a sense to be made precise in Section IV C. There will then effectively be no final conditions at all on the cosmological histories.

As for the choice of the initial boundary conditions, we will examine two instructive examples in the sequel. In order to illustrate how the present sum-over-histories construction can predict semiclassical behaviour in a suitable limit as naturally as sum-over-histories formulations always do, as a first example we consider a single initial localized WheelerDeWitt wave packet $\Psi^{W P}$ which is designed to prefer a particular classical path over all others in the semiclassical limit. In a partition of the minisuperspace histories by classical paths, a steepest descents evaluation of the path integral for the class operators then reveals that the primary contribution to the decoherence functional comes from the class operator corresponding to the coarse-grained class of paths containing the classical path preferred by the initial condition $\Psi^{W P}$.

Next, we shall turn our attention to the semiclassical predictions of the decoherence functional with an initial state of the more traditional WKB form. As is to be expected, under semiclassical coarse grainings such a choice of initial state leads to a distribution of classical trajectories with initial value weighted according to the WKB prefactor, and initial momentum given by the gradient of the phase.

Let us now proceed with the details. 


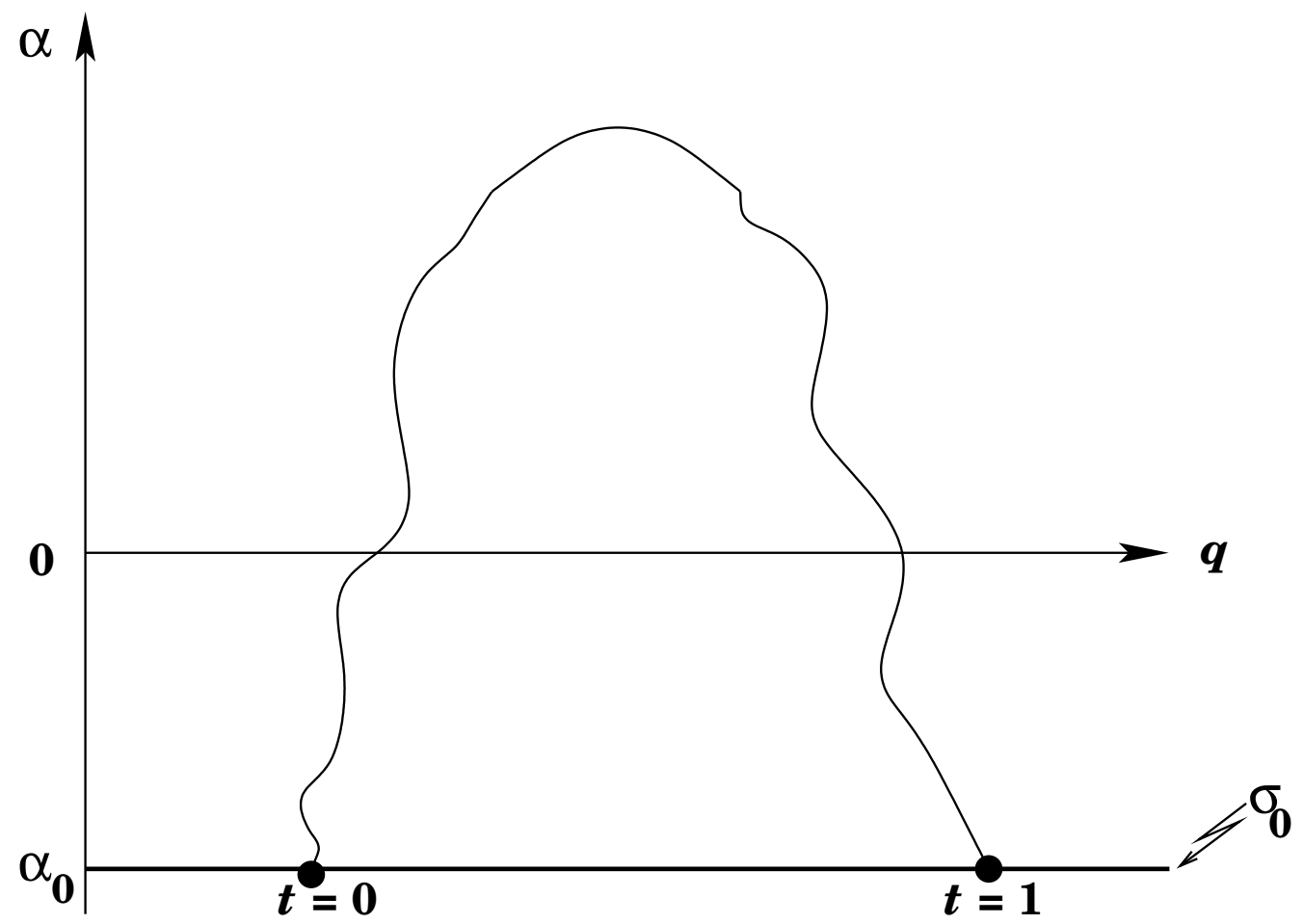

FIG. 1: The minisuperspace of homogeneous cosmological models. The timelike coordinate $q^{0}=\alpha$ and one spacelike coordinate $\left(e . g . q^{1}=\beta_{+}\right)$are plotted. Fine grained histories are paths in this configuration space which begin and end on a surface $\sigma_{0}$ that is spacelike in the Wheeler-DeWitt minisuperspace metric $G_{A B}$ and located at large negative $\alpha\left(=\alpha_{0}\right.$, say), corresponding to a small spatial volume. The restriction to paths which begin and end on $\sigma_{0}$ corresponds to the imposition of a boundary condition that solutions to the Wheeler-DeWitt equation vanish as $\alpha \rightarrow \infty$. The paths all possess $\dot{\alpha}(0)>0$ and $\dot{\alpha}(1)<0$ on $\sigma_{0}$, i.e. "expanding" initial and "contracting" final conditions. The universe expands from a small to a maximum volume and then recontracts.

\section{B. Branch Wave Functions}

First, it is useful to define the "branch wave functions"

$$
\begin{aligned}
\Psi_{h}(q) & =\left\langle q \| C_{h} \mid \Psi\right\rangle \\
& \equiv\left\langle q\left\|C_{h}\right\| q^{\prime}\right\rangle \circ \Psi\left(q^{\prime}\right)
\end{aligned}
$$

These may be regarded as the wave functions corresponding to the initial state, propagated by the restricted propagator (class operator) corresponding to the physical history in question. $^{12}$

The space $\left\{\Psi_{h}(q)\right\}$ of branch wave functions will be denoted $\mathcal{B}$. It depends in an essential way both on the allowed space of initial wave functions $\Psi(q)$, and on the allowed coarse grainings.

${ }^{12}$ It may be helpful to be reminded that the "matrix" appearing in (4.1) should not be complex conjugated when computing the product. 
For arbitrary coarse grainings, it is not immediately evident that $\Psi_{h}(q)$ for $q \in \sigma_{0}$ must be in the space $\mathcal{F}\left(\sigma_{0}\right)$ of functions which fall off rapidly at infinity on $\sigma_{0}$, even when the initial wave functions $\Psi(q)$ are required to be in $\mathcal{F}\left(\sigma_{0}\right)$, as we have done. However, we will take the arguments of Higuchi and Wald [10] as strongly suggestive that initial states in $\mathcal{F}\left(\sigma_{0}\right)$, propagated by the full propagator $\left\langle q^{\prime \prime}\left\|C_{u}\right\| q^{\prime}\right\rangle$ off of $\sigma_{0}$ and then back to $\alpha \rightarrow-\infty$, indeed remain in $\mathcal{F}\left(\sigma_{0}\right)$ when there is a scalar field present. ${ }^{13}$ For the present, we will assume when necessary that attention is restricted to coarse grainings for which all $\Psi_{h}(q) \in \mathcal{F}\left(\sigma_{0}\right)$ when $q \in \sigma_{0}$ i.e. for which $\left.\mathcal{B}\right|_{\sigma_{0}} \subset \mathcal{F}\left(\sigma_{0}\right)$.

\section{Final Indifference}

Boundary conditions of "final indifference" should amount, in essence, to no final boundary condition at all, in the sense that the endpoints of all paths are weighted equally. The final boundary condition then effectively disappears from the decoherence functional, just as it does in ordinary quantum mechanics.

Let us be slightly more precise about how to do this.

What we seek are a complete set of solutions to the Wheeler-DeWitt equation (3.5) which may serve to define a positive "resolution of the identity" in the space of branch wave functions $\mathcal{B}$. By this we mean the following. First, note that if we define

$$
\mathcal{I}\left(q_{2}^{\prime \prime}, q_{1}^{\prime \prime}\right)=\sum_{i} p_{i}^{\prime \prime} \Phi_{i}\left(q_{2}^{\prime \prime}\right) \Phi_{i}^{*}\left(q_{1}^{\prime \prime}\right)
$$

for $q_{1}^{\prime \prime}, q_{2}^{\prime \prime} \in \sigma_{0}$, the decoherence functional (3.9) for the case of a pure initial state $\Psi$ may be written very simply in terms of the branch wave functions (4.1) as

$$
\begin{aligned}
D\left(h, h^{\prime}\right) & =\mathcal{N} \sum_{i} p_{i}^{\prime \prime}\left(\Phi_{i} \circ \Psi_{h}\right)\left(\Phi_{i} \circ \Psi_{h^{\prime}}\right)^{*} \\
& =\mathcal{N} \Psi_{h^{\prime}} \circ \mathcal{I} \circ \Psi_{h} .
\end{aligned}
$$

In order to capture the notion of "final indifference", we next split $\mathcal{B}$ into orthogonal sectors $\mathcal{B}^{ \pm}$on which the Wheeler-DeWitt product $\circ$ is positive or negative definite. ${ }^{14}$ We require for final indifference that the $\Phi_{i}$ be chosen so that

$$
\mathcal{I} \circ \psi^{ \pm}= \pm \psi^{ \pm}
$$

13 The essence of their argument is that for scalar potentials which do not grow exponentially at infinity, the scalar potential term in (3.5) becomes negligible as $\alpha \rightarrow-\infty$ and hence the scalar momentum acts as a conserved mass term in the equation of motion. This means that wave packets "lose energy" upon each bounce off of mixmaster's potential walls, eventually slowing to the point where they move more slowly than the walls recede. Wave packets therefore should become asymptotically "free" as $\alpha \rightarrow-\infty$ inside the potential walls, escaping to infinity more slowly than the receding walls.

14 We are not familiar with an explicit procedure for performing this split of the Bianchi IX minisuperspace in general. (See however $[8,11,43,46]$.) Nonetheless, as argued below, since we only need to evaluate the products appearing in (4.3) at very small volume, the arguments of Higuchi and Wald [10] noted above suggest that the construction we give when the branch wave functions on $\sigma_{0}$ are in $\mathcal{F}\left(\sigma_{0}\right)$ is sufficient for our purposes. 
where $\psi^{ \pm} \in \mathcal{B}^{ \pm}$

Such an identity $\mathcal{I}$ may be explicitly constructed by choosing bases $\left\{\Phi_{i}^{ \pm}\right\}$for $\mathcal{B}^{ \pm}$which are orthogonal in the inner product (3.6),

$$
\Phi_{i}^{ \pm} \circ \Phi_{j}^{ \pm}= \pm \delta_{i j}
$$

and

$$
\Phi_{i}^{ \pm} \circ \Phi_{j}^{\mp}=0
$$

Setting all the $p_{i}^{\prime \prime}=1$, with such a basis we may construct projection operators

$$
\mathcal{I}^{ \pm}\left(q_{2}^{\prime \prime}, q_{1}^{\prime \prime}\right)=\sum_{i} \Phi_{i}^{ \pm}\left(q_{2}^{\prime \prime}\right) \Phi_{i}^{ \pm *}\left(q_{1}^{\prime \prime}\right)
$$

for which

$$
\mathcal{I}^{ \pm}\left(q_{2}^{\prime \prime}, q_{1}^{\prime \prime}\right) \circ \psi\left(q_{1}^{\prime \prime}\right)= \pm \psi^{ \pm}\left(q_{2}^{\prime \prime}\right)
$$

$\mathcal{I}$ is then given by

$$
\mathcal{I}=\mathcal{I}^{+}+\mathcal{I}^{-}
$$

so that

$$
\mathcal{I} \circ \psi=\psi^{+}-\psi^{-}
$$

Explicit calculations require an explicit choice of the $\Phi_{i}^{ \pm}$. Since we are restricting our attention to coarse grainings whose branch wave functions $\Psi_{h}(q)$ lie in $\mathcal{F}\left(\sigma_{0}\right)$, we can implement the split of the space of branch wave functions into $\mathcal{B}^{ \pm}$by choosing the $\left\{\Phi_{i}^{ \pm}\right\}$to be of definite frequency on $\sigma_{0}$ with respect to $\alpha$. That is, relabelling the index $i \rightarrow \vec{p}$,

$$
\frac{\partial}{\partial \alpha} \Phi_{\vec{p}}^{ \pm}(q)=\mp i \omega_{p} \Phi_{\vec{p}}^{ \pm}(q)
$$

For this to be possible, it must be that the Wheeler-DeWitt operator $H$ and $\partial / \partial \alpha$ commute. In fact,

$$
\left[\frac{\partial}{\partial \alpha}, H\right] f\left(\alpha, \beta_{ \pm}, \phi\right)=4 e^{4 \alpha} V_{\beta}\left(\beta_{ \pm}\right) f\left(\alpha, \beta_{ \pm}, \phi\right)+6 e^{6 \alpha} V_{\phi}(\phi) f\left(\alpha, \beta_{ \pm}, \phi\right)
$$

so that $\partial / \partial \alpha$ and $H$ approximately commute everywhere on surfaces of large negative $\alpha$ so long as they act in the space of functions $\mathcal{F}\left(\sigma_{0}\right)$ for which $V_{\beta} f$ and $V_{\phi} f$ remain bounded. Since $V_{\beta}$ and $V_{\phi}$ are both bounded below, this is equivalent to the condition that $V f \approx 0$ on $\sigma_{0}$ - or more precisely, that $e^{-4 \alpha} V f$ is bounded - whence our choice of boundary conditions on the allowed solutions to the constraint in Section III A 3.

The explicit form of the $\Phi_{\vec{p}}^{ \pm}$is not difficult to find. It is clear from (2.9) that on $\sigma_{0}$ there are large regions near the origin in $\vec{q}$-space for which the potential $V$ is totally negligible so long as $V_{\phi}$ remains bounded. In fact, it is a standard part of the lore of mixmaster cosmologies $^{15}$ that $V_{a}$, the anisotropy potential for Bianchi IX - see below (2.7) - is wellapproximated as $\alpha \rightarrow-\infty$ by a triangular set of potential walls at $\alpha=-\left(\beta_{+} \pm \sqrt{3} \beta_{-}\right)$and

15 [47] are useful additions to the literature already cited. The discussion in section IV of [10] provides an extremely useful formulation for general type A Bianchi models; see also [29] for Bianchi IX. 
$\alpha=2 \beta_{+}$. Well inside these walls, we may take $V$ to be essentially zero for reasonable $V_{\phi}{ }^{16}$ Near the origin of the spacelike surface $\sigma_{0}$, the $\Phi_{\vec{p}}^{ \pm}(q)$ can then be taken to have the form

$$
\Phi_{\vec{p}}^{ \pm}(q)=\frac{1}{\sqrt{(2 \pi)^{n} 2 \omega_{p}}} e^{\mp i \omega_{p} \alpha} e^{i \vec{p} \cdot \vec{q}}
$$

where $\omega_{p}^{2}=\vec{p}^{2}$. In the asymptotic region where the potential $V$ is not negligible we may choose to maintain either (3.5) or (4.11), since our branch wave functions are by design essentially zero there. Should we choose to adhere strictly to the requirement (3.5) everywhere on $\sigma_{0}$, the $\Phi_{\vec{p}}^{ \pm}$will of course be more complicated in the asymptotic region. Practically, however, it is usually easiest to retain the form (4.13) everywhere. So long as we restrict attention to initial states and coarse-grainings for which $\left.\mathcal{B}\right|_{\sigma_{0}} \subset \mathcal{F}\left(\sigma_{0}\right)$ the distinction will be practically irrelevant and the explicit form (4.13) can be used in calculating the decoherence functional.

The basis of functions (4.13) are orthogonal in the appropriate sense,

$$
\Phi_{\vec{p}}^{ \pm} \circ \Phi_{\vec{p}^{\prime}}^{ \pm}= \pm \delta_{\vec{p} \vec{p}^{\prime}}^{(n)}
$$

and

$$
\Phi_{\vec{p}}^{ \pm} \circ \Phi_{\vec{p}^{\prime}}^{\mp}=0 .
$$

( $n$ is the number of "spatial" minisuperspace degrees of freedom - three, in the present example, $\beta_{ \pm}$and $\phi$.) The projections $\mathcal{I}^{ \pm}$of (4.7) may then be explicitly constructed as $\left(\right.$ taking $\left.\sum_{i} p_{i}^{\prime \prime} \rightarrow \int d^{n} p\right)$

$$
\mathcal{I}^{ \pm}\left(q_{2}^{\prime \prime}, q_{1}^{\prime \prime}\right)=\int d^{n} p \Phi_{\vec{p}}^{ \pm}\left(q_{2}^{\prime \prime}\right) \Phi_{\vec{p}}^{ \pm *}\left(q_{1}^{\prime \prime}\right)
$$

Finally, we note that the orthonormal bases $\left\{\Phi_{i}^{ \pm}\right\}$are also useful to represent the initial condition $\Psi$. Indeed, solutions to the Wheeler-DeWitt equation in $\mathcal{F}\left(\sigma_{o}\right)$ may be written as

$$
\begin{aligned}
\Psi & =\sum_{i} c_{i}^{+} \Phi_{i}^{+}+\sum_{i} c_{i}^{-} \Phi_{i}^{-} \\
& =\Psi^{+}+\Psi^{-}
\end{aligned}
$$

where

$$
c_{i}^{ \pm}= \pm \Phi_{i}^{ \pm} \circ \Psi
$$

For the particular choice of basis (4.13) for $\mathcal{F}\left(\sigma_{o}\right)$, this looks like

$$
\Psi(q)=\int d^{n} p\left\{\tilde{\Psi}^{+}(\vec{p}) \Phi_{\vec{p}}^{+}(q)+\tilde{\Psi}^{-}(\vec{p}) \Phi_{\vec{p}}^{-}(q)\right\}
$$

where

$$
\tilde{\Psi}^{ \pm}(\vec{p})= \pm \Phi_{\vec{p}}^{ \pm} \circ \Psi
$$

on $\sigma_{0}$.

${ }^{16}$ Kuchař has shown [48] that this "asymptotic freedom" of the gravitational potential $V$ is a generic feature of superspace, and is not special to homogeneous models. 


\section{The Wheeler-DeWitt Product and the Decoherence Functional}

With the resolution of the identity $\mathcal{I}$ in hand, we define the positive definite WheelerDeWitt product $\bullet$ by

$$
\begin{aligned}
\psi \bullet \psi & =\psi \circ \mathcal{I} \circ \psi \\
& =\psi^{+} \circ \psi^{+}-\psi^{-} \circ \psi^{-} .
\end{aligned}
$$

In terms of this positive product, ${ }^{17}$ the decoherence functional may be written very simply as

$$
D\left(h, h^{\prime}\right)=\mathcal{N} \Psi_{h^{\prime}} \bullet \Psi_{h},
$$

where $\mathcal{N}$ is $\left(\Psi_{u} \bullet \Psi_{u}\right)^{-1}$. (4.22) is the form of the decoherence functional we will use for the remainder of the discussion. The generalization of this formula to the case of an impure set of initial wave functions $\left\{\Psi_{i}, p_{i}^{\prime}\right\}$ is obvious.

\section{E. Classical Initial Conditions}

Because we will be interested in initial conditions and coarse grainings which distinguish classical behaviour, it is useful to discuss the conditions necessary to specify classical solutions.

The classical equations of motion - the reduced Einstein equations - resulting from the variation $\delta S / \delta q=0$ of the Lagrangian action appearing in (3.17a) are, in the proper time gauge (3.13),

$$
\frac{1}{2 N} \frac{d^{2} q_{A}}{d t^{2}}=-N \nabla_{A} V
$$

along with the Lagrangian version of the constraint $\delta S / \delta N=0$ :

$$
\frac{1}{4 N} \frac{d q^{A}}{d t} \frac{d q_{A}}{d t}+N V=0
$$

Fixing a classical solution starting on $\sigma_{0}$ therefore requires two pieces of minisuperspace data for each $q^{A}$, subject to the constraint (4.24). The initial data cannot be chosen arbitrarily even if $N$ is regarded as an unknown to be determined by (4.24): for arbitrary initial data there is no guarantee that there exists a real constant $N$ that can satisfy the constraint $\dot{\alpha}_{0}^{2}=\dot{\vec{q}}_{0}^{2}+4 N^{2} V\left(q_{0}\right)$. The initial data must be chosen so that $\dot{\alpha}_{0}^{2}-\dot{\vec{q}}_{0}^{2}$ has the same sign as $V\left(q_{0}\right)$. The $N$ thus fixed by (4.24) will then appear as a parameter in the classical solution emanating from $\sigma_{0}$ specified by the initial data $\left(q_{0}, \dot{q}_{0}\right)$. (Of course, a reparametrization of the time $t$ that preserves the proper time gauge will yield a different value for $N$.)

\footnotetext{
${ }^{17}$ Indeed, up to technical details, we expect the choices we have made are in their effect equivalent to employing the "Rieffel induced" inner product to construct the decoherence functional; see [28, 43, 44] and references therein for further discussion. (4.22), employing the Rieffel induced product, is thus a natural alternative definition of the decoherence functional that automatically incorporates a notion of "final indifference." More generally, note that (4.3) defines a positive product on the space of branch wave functions. When the $\left\{\Phi_{i}\right\}$ constitute a complete set and all the $p_{i}^{\prime \prime} \neq 0$ this is a genuine non-degenerate inner product. One may thus regard the specification of "final indifference" as equivalent to the problem of defining an inner product on the space of branch wave functions.
} 


\section{F. Quantum Initial States: Examples}

We choose our first example initial state $\Psi^{W P}$ to be a positive frequency solution to (3.5) that is localised well within mixmaster's potential walls on the initial surface, and also away from regions of very large $V_{\phi}$. Such an initial state will prefer a particular classical path and predict approximately classical behaviour along the corresponding classical solution.

$\Psi^{W P}$ by assumption solves (3.5) with $V \approx 0$ and is localized near some $\vec{q}_{0}$ on $\sigma_{0}$. It may then be represented as

$$
\begin{aligned}
\Psi^{W P}(q) & =\int d^{3} p \tilde{\Psi}^{W P+}(\vec{p}) \Phi_{\vec{p}}^{+}\left(q-q_{0}\right) \\
& =\int \frac{d^{3} p}{\sqrt{(2 \pi)^{3}}} \frac{1}{\sqrt{2 \omega_{p}}} e^{-i \omega_{p}\left(\alpha-\alpha_{0}\right)} e^{i \vec{p} \cdot\left(\vec{q}-\vec{q}_{0}\right)} \tilde{\Psi}^{W P+}(\vec{p})
\end{aligned}
$$

for some $\tilde{\Psi}^{W P+}(\vec{p})$, where of course $\omega_{p}=|\vec{p}|$. Taking $\omega_{p}^{-1 / 2} \tilde{\Psi}^{W P+}(\vec{p})$ to be a Gaussian centered around some $\vec{p}_{0}$, for instance, yields a $\Psi^{W P}(q)$ localized on $\sigma_{0}$ about $\left(\vec{q}_{0}, \vec{p}_{0}\right)$ to the greatest extent consistent with the uncertainty principle.

Alternatively, we could consider an initial state of WKB form,

$$
\Psi^{W K B}(q)=A(q) e^{i W(q)}
$$

where $A(q)$ is in an appropriate sense slowly varying relative to $W(q)$. Approximate calculations of the wave functions corresponding to the various proposals for the initial condition of the universe tend to have components of this form. We also require that $A(q)$ be of compact support on $\sigma_{0}$ so as to ensure that $\Psi_{0} \in \mathcal{F}\left(\sigma_{0}\right)$, and that $W(q)$ be "positive frequency" in the sense that $\partial W / \partial \alpha<0$ on $\sigma_{0}$. (This will turn out to correspond to an "expanding" initial condition on the paths this wave function defines.)

We will examine both of these choices in the sequel. First, however, we shall move on to discuss the category of coarse grainings defining approximately classical behaviour that we shall consider.

\section{APPROXIMATE CLASSICALITY}

In this section, we apply the decoherence functional (4.22) for $\Lambda=0$, Bianchi IX cosmologies to coarse grainings which distinguish between those paths in minisuperspace which behave (semi-) classically, and those which do not. We find that for suitable choices of the initial condition $\Psi$, and for a suitable class of definitions of the semiclassical coarse grainings, the universe is predicted to behave classically with probability near one. In particular, $\Lambda=0$ Bianchi IX cosmologies are predicted quantum-mechanically to recollapse just as they do classically [29]. This is satisfying, not least because of the surprising prediction of Wald's $[10,26]$ rigorous canonical quantization of Bianchi IX that closed quantum universes do not recollapse in a classical fashion. ${ }^{18}$ (For another discussion of difficulties interpreting wave functions for classically recontracting cosmologies, see [49].)

18 The trouble is rooted in the fact that Wald's quantization essentially employs the volume of the universe (rather, $\alpha$ ) as a "time" variable in the canonical quantization procedure. With this parameterization there is no way to construct minisuperspace wave packets which follow an approximately classical trajectory 
We begin with a simple example to illustrate the general procedure we have in mind, then go on in the subsequent sections to explore semiclassical coarse-grainings and the corresponding class operators and branch wave functions in greater detail.

\section{A. Coarse-Graining by a Single Trajectory}

As a particularly simple example of a semiclassical coarse-graining, ask whether the model universe follows a particular trajectory in minisuperspace $q_{c l}$. To this end introduce the Euclidean distance on superspace and define a region around the curve $q_{c l}$ - say a tube $T$ of radius $\delta$. (See Figure $2 .{ }^{19}$ ) We partition the paths $q(t)$ by whether they lie entirely in $T$ or not, labelling the corresponding classes $c_{T}$ and $c_{\bar{T}}$.

It is plausible that when $\delta$ is sufficiently large the path integral in $(3.17 \mathrm{~b})$ can be done by the stationary phase approximation to find

$$
\begin{aligned}
&\left\langle q^{\prime \prime}\left\|C_{T}\right\| q^{\prime}\right\rangle \approx \begin{cases}\Delta\left(q^{\prime \prime}, q^{\prime}\right) e^{i S_{c l}\left(q^{\prime \prime}, q^{\prime}\right)} & q^{\prime}, q^{\prime \prime} \in T \\
0 & \text { else }\end{cases} \\
&\left\langle q^{\prime \prime}\left\|C_{\bar{T}}\right\| q^{\prime}\right\rangle \approx \begin{cases}\Delta\left(q^{\prime \prime}, q^{\prime}\right) e^{i S_{c l}\left(q^{\prime \prime}, q^{\prime}\right)} & q^{\prime} \text { or } q^{\prime \prime} \notin T \\
0 & q^{\prime}, q^{\prime \prime} \in T\end{cases}
\end{aligned}
$$

so that $C_{T}+C_{\bar{T}}=C_{u}$ as required by (3.3), where $C_{u}$ is the full, unrestricted propagator. Here $\Delta$ is the usual semiclassical prefactor and $S_{c l}$ is the action evaluated on the classical path connecting $q^{\prime}$ to $q^{\prime \prime}$. (See Section V C for further details.)

Now consider the special case of an initial condition $\Psi\left(q^{\prime}\right)$ whose center moves along $q_{c l}(t)$. Given (5.1),

$$
\begin{aligned}
\Psi_{T}\left(q^{\prime \prime}\right) & =\left\langle q^{\prime \prime}\left\|C_{T}\right\| q^{\prime}\right\rangle \circ \Psi\left(q^{\prime}\right) \\
& \approx \begin{cases}\Delta\left(q^{\prime \prime}, q^{\prime}\right) e^{i S_{c l}\left(q^{\prime \prime}, q^{\prime}\right)} \circ \Psi\left(q^{\prime}\right) & q^{\prime \prime} \in T \\
0 & \text { else }\end{cases} \\
\Psi_{\bar{T}}\left(q^{\prime \prime}\right) & \approx 0
\end{aligned}
$$

(See Figure 3.) Thus we expect according to (3.1) and (4.22) the probability for $\Psi_{T}$ to be approximately 1 and the probability for $\Psi_{\bar{T}}$ to be approximately 0 . For these special choices

through both expanding and recollapsing phases. Moreover, there appears no sensible way to define an operator momentum conjugate to $\alpha$ (which corresponds physically to the expansion rate) that can have both positive and negative eigenvalues [9]. In other words, this quantization seems to have an "arrow of time" that precludes the construction of states which follow the full course of a classical evolution. Instead, time appears to "freeze" as the universe approaches its classical maximum size [10], in the sense that physical variables cease to evolve.

${ }^{19}$ Note that strictly speaking, $T$ must not intersect $\sigma_{0}$ in order that the coarse graining does not restrict paths on the initial surface. This was necessary in Section III A 3 to guarantee the independence of the decoherence functional from the choice of initial surface. We may choose either to give up this independence for coarse-grainings of this kind - not a surprising or onerous restriction for coarse-grainings which explicitly restrict paths on the initial surface - or end the tube $T$ at a volume $\alpha_{*}$ larger than $\alpha_{0}$, as in the figure. When $\alpha_{*}$ is close to $\alpha_{0}$, or more generally for systems which are not chaotic, we expect these approaches to be practially equivalent. 


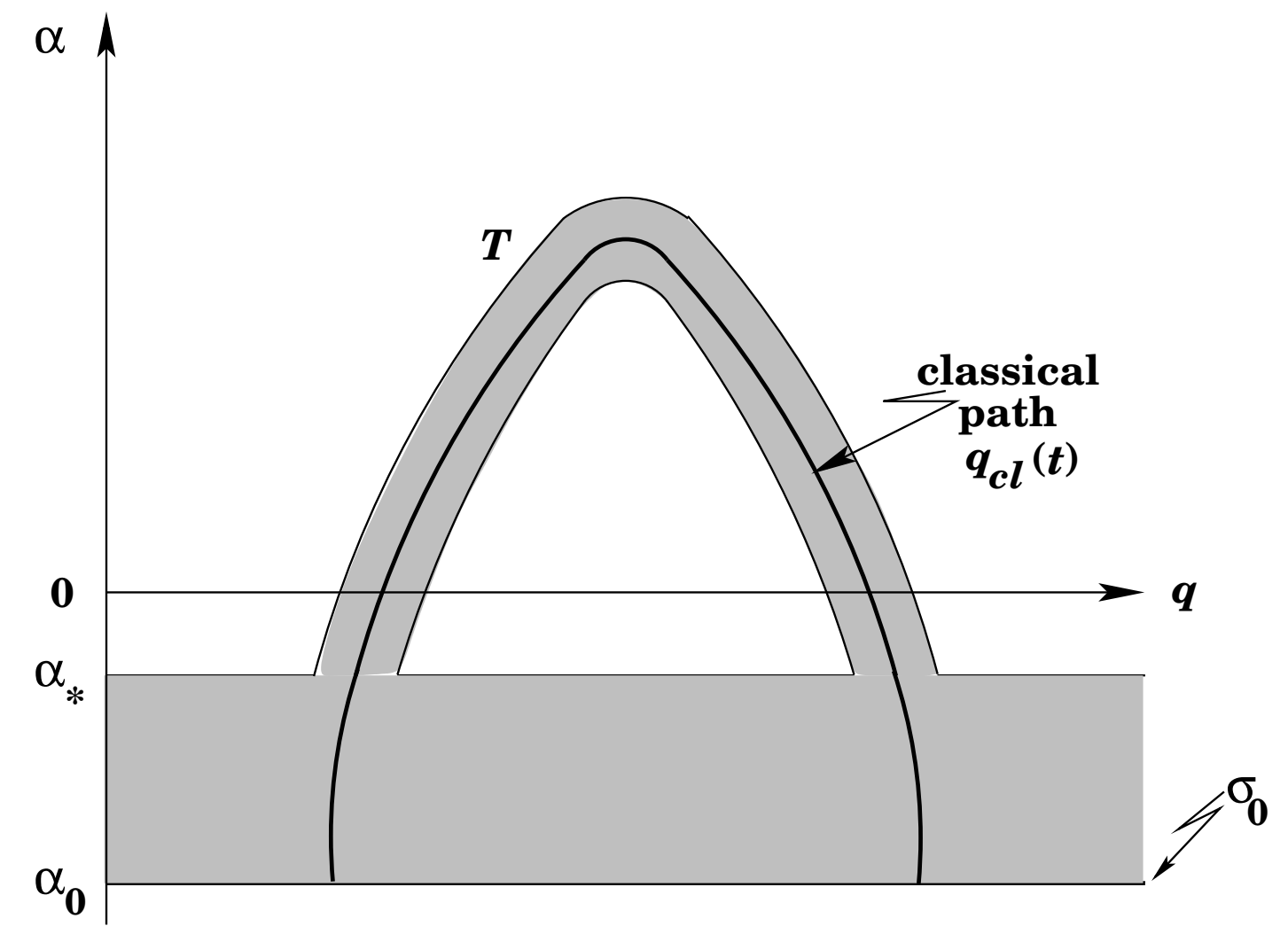

FIG. 2: Schematic representation of a course-graining defining classical behaviour. The shaded region $T$ illustrated in the figure consists of all configuration space for $\alpha<\alpha_{*}$, and for $\alpha>\alpha_{*}$, a tube surrounding a trajectory $q_{c l}(t)$ that obeys the classical equations of motion. The fine grained cosmological histories can be divided into the class $c_{T}$ that lie entirely inside $T$, and the class $c_{\bar{T}}$ that are sometimes outside of it (possible only for $\alpha>\alpha_{*}$.) The probability of $c_{T}$ is the probability that the evolution of the universe is approximately the classical evolution $q_{c l}(t)$ for $\alpha>\alpha_{*}$.

of coarse-graining and initial condition, therefore, the prediction is that the model universe "behaves semiclassically" over the whole of its evolution from near the big bang to the big crunch.

\section{B. Coarse Grainings by Classical Paths}

The coarse-graining of the previous section asks only whether the evolution of the universe is close to a particular $q_{c l}(t)$. More generally we can ask for the probability that it evolves close to any classical path. In this section we discuss the corresponding coarse-grainings.

Coarse-grainings that can serve to distinguish classical behaviour can be characterized generally according to whether a minisuperspace trajectory is "close to" a classical trajectory in some appropriate sense. Because a classical 4-geometry is fixed by a solution $z(t)=$ $\left(q^{A}(t), N\right)$ to the proper time gauge Einstein equations (4.23) and (4.24), we say that $z(t)$ is "approximately classical" when there exists a solution $z_{c l}(t)$ to Einstein's equations for which

$$
F\left[z(t), z_{c l}(t)\right]<1
$$




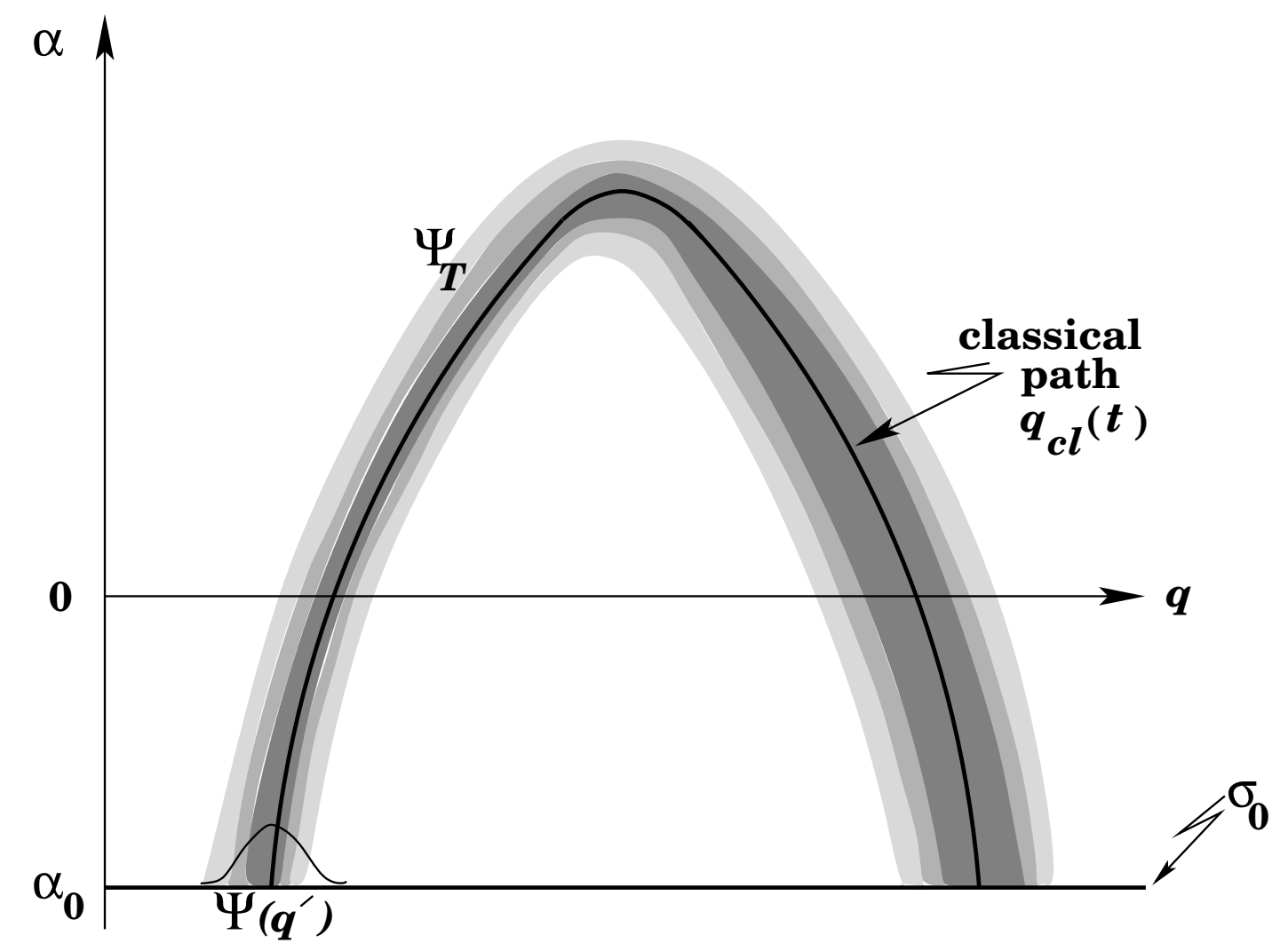

FIG. 3: Schematic representation of the region of support of the semiclassical branch wave function $\Psi_{T}\left(q^{\prime \prime}\right)$ corresponding to the class of paths $c_{T}$ for a localized initial condition $\Psi\left(q^{\prime}\right) . \Psi_{T}\left(q^{\prime \prime}\right)$ is concentrated around the classical trajectory $q_{c l}(t)$ preferred by the initial condition.

where $F[\cdot, \cdot]$ is a reparametrization invariant distance functional on the extended space of paths $\mathcal{C}_{\text {ext }} . F$ thereby partitions the space of paths into the class $c_{c l}$ of those which behave classically, according to the standard $F$, and the class which do not, $c_{q m}$. An example of such a functional is the Euclidean distance on superspace employed above, which can be rewritten in the form (5.3) simply by dividing by $\delta$.

More refined coarse grainings are also possible. It is naturally of interest to know to which path(s) $z(t)$ may be close. Various strategies are possible; we describe one here that will be employed in the sequel.

First, partition the classical trajectories into classes $c_{i j}$, possibly by ranges $\left\{\Delta_{i}^{\prime}, \Delta_{j}^{\prime \prime}\right\}$ of initial and final position on $\sigma_{0}$, or by ranges of initial position and momentum $\left\{\Delta_{i}^{\prime}, \Gamma_{j}^{\prime}\right\}$. The former might be of interest when we want to make predictions about universes which, say, begin and end with low anisotropy; the latter, when we are more interested in comparing universes with differing initial states. (Either way, the classical solutions provide a natural mapping $\left\{\Delta_{i}^{\prime}, \Delta_{j}^{\prime \prime}\right\} \leftrightarrow\left\{\Delta_{i}^{\prime}, \Gamma_{j}^{\prime}\right\}$ for a given $\Delta_{j}^{\prime \prime}$ or $\Gamma_{j}^{\prime}$.) Next, for each quantum path, compute

$$
\inf _{z_{c l}(t)} F\left[z(t), z_{c l}(t)\right]
$$

say, by minimizing with respect to the initial conditions of the classical paths. If this number is greater than one, then $z(t) \in c_{q m}$. Otherwise, collect $z(t)$ into the same class $c_{i j}$ as the 
$z_{c l}(t)$ which obtains the minimum. ${ }^{20}$

A particular choice of semiclassical coarse-graining entails the selection of a particular distance functional $F$. However, we expect reasonable characterizations of semi-classical behaviour to display a certain robustness and a consequent insensitivity to the details of the definition of the coarse-graining (choice of $F$ ) for most practical applications. Nevertheless, we can make a few general observations about what constitutes an acceptable classical distance functional on $\mathcal{C}_{\text {ext }}$.

First, $F$ must not be such as to imply that paths obeying (5.3) possess finite action. This is a consequence of the well known [50] fact that paths of finite action contribute zero measure to functional integrals. Instead, it is the small but non-differentiable fluctuations about the classical stationary points which dominate the integral. In this sense, the coarse graining must not be "too small", or in other words must not be so refined as to exclude the essential contributions of the truly quantum paths in the neighborhood of the classical trajectories. In particular, coarse grainings which imply that paths obeying (5.3) are differentiable are excluded from consideration. (Since the potential $V$ is finite, the action will be finite if the kinetic terms are.) This condition is necessary in order that we can make sense of the stationary phase approximation, and in particular argue that the values of the functional integrals are indeed well approximated by their stationary phase values when integrating only over paths satisfying (5.3).

This stationary-phase condition may be satisfied by admitting as semi-classical coarse grainings those which include all paths nearby each classical trajectory with a minimum Euclidean distance scale set by $1 / \sqrt{\lambda}$, where the $\lambda$ are the eigenvalues of $\delta^{2} S\left[q_{c l}(t)\right] / \delta q^{A}\left(t^{\prime}\right) \delta q^{B}\left(t^{\prime \prime}\right)$.

Second, the coarse graining must not be "too large", in the sense that paths in the class $c_{c l}$ of approximately classical paths must be meaningfully distinct from those in the complementary class $c_{q m}$. The criteria applied to make this distinction will in general be particular to individual physical problems in which a notion of approximate classicality is to be applied. Nonetheless, for most semi-classical considerations, the scale of such coarsegrainings will typically be far coarser than the lower limits implied by the considerations of the previous paragraphs.

Finally, it is a generic feature of quantum mechanics that localized states spread over time. In the absence of a stabilization mechanism such as environmental decoherence through interaction with other degrees of freedom in a system [35-37, 51-53], even a wave packet whose center follows a classical trajectory may eventually spread sufficiently that its behaviour is unrecognizably classical. While such stabilization mechanisms are widespread in the real universe (as opposed to toy models with few degrees of freedom), the phenomenon of wave packet spreading may be a relevant factor in determining whether a particular choice of coarse-graining is meaningfully "semi-classical" for a given physical problem.

There are, of course, many choices for the distance functional $F$ that will satisfy the general requirements laid out above $[1,36]$. However, in accordance with the general ex-

${ }^{20}$ For reasonable $F$ 's, this should be a genuine partition of the paths. In those rare cases for which there are $z_{c l}(t)$ in different $c_{i j}$ which yield the same minimum, additional criteria must be selected and applied to complete the partition. Similarly, if there are qualitatively distinct classical paths connecting points in $\Delta_{i}^{\prime}$ to $\Delta_{j}^{\prime \prime}$ - in the case where there are multiple classical paths connecting a single pair of points, for example - then it may under some circumstances be desirable to further partition the class $c_{i j}$. We shall take this as understood without attempting to incorporate it into our notation explicitly. 
pectation that predictions concerning semiclassical behaviour will be relatively insensitive to the details of the choice of $F$, the approximate calculations given in the sequel will be sufficiently coarse that a detailed specification will not be needed. When necessary, we will typically have in mind something like the Euclidean distance on superspace employed in Section V A. (A more careful assessment of suitable choices for the distance functional $F$ in the context of ordinary quantum mechanics will be given elsewhere [54].)

\section{Semiclassical Propagator}

Let us now proceed with the approximate evaluation of the class operators for the general category of semiclassical coarse-grainings described above.

In the $\dot{N}=0$ (proper time) gauge, the class operators $[c f .(3.16-3.17)]$ read

$$
\left\langle q^{\prime \prime}\left\|C_{h}\right\| q^{\prime}\right\rangle=\int_{c_{h}} d N_{\geq 0} \int_{c_{h}} \delta q_{q^{\prime}}^{q^{\prime \prime}} \exp \left(i \int_{0}^{1} d t\left[\frac{1}{4 N} G_{A B} \dot{q}^{A} \dot{q}^{B}-N V\right]\right) .
$$

The integral is over all paths from $q^{\prime}$ to $q^{\prime \prime}$ in the class $c_{h}$. It is to be noted that, strictly speaking, the restricted propagator $\left\langle q^{\prime \prime}\left\|C_{h}\right\| q^{\prime}\right\rangle$ need only be defined for $q^{\prime}$ and $q^{\prime \prime}$ in $\sigma_{0}$. $c_{h}$, however, defines a (possibly empty) class of paths passing through any $q^{\prime \prime}$ in minisuperspace, and when $q^{\prime \prime}$ is not in $\sigma_{0}$ the integral over these paths is what is meant by the path integral in (5.5). The set of points in minisuperspace for which this class is non-empty will be denoted $T_{h}$, which will therefore be the region of support in $q^{\prime \prime}$ of the class operator (5.5) in minisuperspace.

Let us begin by approximately evaluating the full propagator, the case in which $c_{h}$ in $(5.5)$ happens to be the class of all paths $c_{u}$ from $q^{\prime}$ to $q^{\prime \prime}$. A steepest descents approximation to the functional integral over $q$ gives

$$
\left\langle q^{\prime \prime}\left\|C_{u}\right\| q^{\prime}\right\rangle=\int_{0}^{\infty} d N \Delta\left(q^{\prime \prime}, q^{\prime} ; N\right) e^{i S_{c l}\left(q^{\prime \prime}, q^{\prime} ; N\right)},
$$

where $S_{c l}\left(q^{\prime \prime}, q^{\prime} ; N\right)$ is the action evaluated for the classical path $q_{c l}\left(q^{\prime \prime}, q^{\prime}: N\right)$ - solution to (4.23) with the given value of $N$ - connecting $q^{\prime}$ to $q^{\prime \prime}$, and

$$
\begin{aligned}
\Delta^{-2}\left(q^{\prime \prime}, q^{\prime} ; N\right) & \propto \operatorname{det} \frac{\delta^{2} S\left[q_{c l}(t)\right]}{\delta q^{A}\left(t^{\prime}\right) \delta q^{B}\left(t^{\prime \prime}\right)} \\
& \propto \operatorname{det}\left[\frac{1}{2 N} G_{A B} \frac{d^{2}}{d t^{2}}+N \nabla_{A} \nabla_{B} V\left(q_{c l}\right)\right],
\end{aligned}
$$

dropping a multiplicative factor of $\operatorname{det}\left[-i \delta\left(t-t^{\prime}\right)\right]$ in the last line that will cancel in the decoherence functional. Similarly, neglecting ${ }^{21}$ the $N$ dependence of $\Delta\left(q^{\prime \prime}, q^{\prime} ; N\right)$ in $(5.6)$, the stationary phase approximation picks out the "zero energy" path(s) in (5.6) obeying the constraint (4.24). Thus,

$$
\left\langle q^{\prime \prime}\left\|C_{u}\right\| q^{\prime}\right\rangle \approx \Delta\left(q^{\prime \prime}, q^{\prime}\right) e^{i S_{c l}\left(q^{\prime \prime}, q^{\prime}\right)}
$$

${ }^{21}$ Heuristically, if we exponentiate the determinant via $\ln \operatorname{det} \mathcal{O}=\operatorname{tr} \ln \mathcal{O}$, the $N$ dependence of the semiclassical prefactor $\Delta\left(q^{\prime \prime}, q^{\prime} ; N\right)$ is only logarithmic, as compared with that of $S_{c l}\left(q^{\prime \prime}, q^{\prime} ; N\right)$. 
where $N$ now has its classical dependence $N\left(q^{\prime \prime}, q^{\prime}\right)$ on the endpoints of the path given by substituting $q_{c l}\left(q^{\prime \prime}, q^{\prime} ; N\right)$ into the constraint (4.24). ${ }^{22}$ When there is more than one classical path connecting $q^{\prime}$ to $q^{\prime \prime}$, then there will be a sum over such paths in (5.8).

According to the famous Van Vleck formula [55, 56], $\Delta\left(q^{\prime \prime}, q^{\prime}\right)$ may be evaluated as

$$
\Delta\left(q^{\prime \prime}, q^{\prime}\right)=\left(\frac{1}{2 \pi i} \frac{\partial^{2} S_{c l}\left(q^{\prime \prime}, q^{\prime}\right)}{\partial N_{c l}^{2}}\right)^{-\frac{1}{2}}\left(\frac{1}{2 \pi i \hbar}\right)^{\frac{d}{2}}\left|\operatorname{det} \frac{\partial^{2} S_{c l}\left(q^{\prime \prime}, q^{\prime}\right)}{\partial q^{\prime \prime} \partial q^{\prime}}\right|^{\frac{1}{2}} e^{-\frac{i \nu \pi}{2}},
$$

where $d=n+1$ is the number of minisuperspace degrees of freedom -4 , in the present example, $\alpha, \beta_{ \pm}$, and $\phi-$ and $\nu$ is the "Maslov index" of the path $q_{c l}(t)$ [55-57]. Roughly speaking, it counts the number of negative eigenvalues of $\delta^{2} S\left[q_{c l}(t)\right] / \delta q^{A}\left(t^{\prime}\right) \delta q^{B}\left(t^{\prime \prime}\right)$ along the trajectory $q_{c l}(t)$. The first factor arises of course from the ordinary stationary phase integral over $N$.

In subsequent sections we will go on to discuss the prediction of semiclassical evolution of the universe for various choices of initial state. For this purpose it will be helpful to close this section with the observation that, as a consequence of the choice of positive range for the lapse $N$ and the boundary conditions on the ends of the paths on $\sigma_{0}, \Delta^{*}\left(q^{\prime \prime}, q^{\prime}\right) e^{-i S_{c l}\left(q^{\prime \prime}, q^{\prime}\right)}$, considered as a function of $\alpha^{\prime}$, is positive frequency in $\alpha^{\prime}$. Recalling that the propagator in (3.8) or (4.1) is not complex-conjugated when forming the indicated products, this means that $\Delta\left(q^{\prime \prime}, q^{\prime}\right) e^{i S_{c l}\left(q^{\prime \prime}, q^{\prime}\right)}$ overlaps only with the positive frequency part of the initial wave function $\Psi . \Delta\left(q^{\prime \prime}, q^{\prime}\right) e^{i S_{c l}\left(q^{\prime \prime}, q^{\prime}\right)}$ is similarly negative frequency in $\alpha^{\prime \prime}$, so that only the negative frequency pieces of the final boundary conditions $\Phi_{i}$ contribute. This is to be expected, as the full propagator given by the path integral (3.14) over all paths and with a positive range for the lapse is in essence ( $i$ times) the Feynman propagator for this minisuperspace theory [39].

To see why it is true that $\Delta^{*}\left(q^{\prime \prime}, q^{\prime}\right) e^{-i S_{c l}\left(q^{\prime \prime}, q^{\prime}\right)}$ is positive frequency in $\alpha^{\prime}$, we shall show that its Fourier transform only has support for positive frequencies $\omega^{\prime}$. That is, consider

$$
\int d \alpha^{\prime} e^{i \omega^{\prime} \alpha^{\prime}} \Delta^{*}\left(q^{\prime \prime}, q^{\prime}\right) e^{-i S_{c l}\left(q^{\prime \prime}, q^{\prime}\right)}
$$

Again neglect the $\alpha^{\prime}$ dependence of $\Delta^{*}\left(q^{\prime \prime}, q^{\prime}\right)$ as logarithmically slower than that of $S_{c l}$. The largest contribution to the integral comes from the point of stationary phase

$$
\omega^{\prime}=\frac{\partial S_{c l}}{\partial \alpha^{\prime}}
$$

Considering the action appearing in (5.5) as a functional of classical paths, it is a straightforward matter to verify that under variations of the endpoints of those classical paths

$$
\delta S_{c l}=\left.\frac{\dot{q}_{c l}^{A}}{2 N_{c l}} \delta q_{A}\right|_{0} ^{1}
$$

\footnotetext{
${ }^{22}$ Strictly speaking, it is the time integral of (4.24) that is the constraint that emerges from the stationary phase approximation to the integral over $N$, viz. $\frac{\partial}{\partial N} S\left[q_{c l}\left(q^{\prime \prime}, q^{\prime} ; N\right)\right]=0$. However, the left hand side of (4.24) is the "energy integral" of solutions to $\delta S / \delta q=0$ (i.e. (4.23)), and hence is actually constant, so that the integral sign may be dropped.
} 
where $q_{c l}^{A}(t)$ is the classical path joining $q^{\prime}=q_{c l}(0)$ to $q^{\prime \prime}=q_{c l}(1)$. Thus

$$
\frac{\partial S_{c l}}{\partial \alpha^{\prime}}=\frac{\dot{\alpha}_{c l}(0)}{2 N_{c l}}
$$

As we have chosen $N$ to be positive, and $\dot{\alpha}_{c l}(0)$ is positive for all classical paths ${ }^{23}$ that begin on our boundary surface of large negative $\alpha$, we see that only a positive $\omega^{\prime}$ contributes significantly to $(5.10)$, so that $\Delta^{*}\left(q^{\prime \prime}, q^{\prime}\right) e^{-i S_{c l}\left(q^{\prime \prime}, q^{\prime}\right)}$ is positive frequency in $\alpha^{\prime}$. Similarly $\Delta\left(q^{\prime \prime}, q^{\prime}\right) e^{+i S_{c l}\left(q^{\prime \prime}, q^{\prime}\right)}$ is negative frequency in $\alpha^{\prime \prime}$. This means that $\Delta\left(q^{\prime \prime}, q^{\prime}\right) e^{+i S_{c l}\left(q^{\prime \prime}, q^{\prime}\right)}$ is negative frequency in both $\alpha^{\prime}$ and $\alpha^{\prime \prime}$. (This is a consequence of the fact that the classical paths both begin and end on the same surface, so that $\dot{\alpha}_{c l}(0)>0$ while $\dot{\alpha}_{c l}(1)<0$ on $\sigma_{0}$. This difference cancels the sign difference between the endpoints that arises in the variation of the action (5.12).) As noted, the semiclassical class operator $\Delta\left(q^{\prime \prime}, q^{\prime}\right) e^{+i S_{c l}\left(q^{\prime \prime}, q^{\prime}\right)}$ therefore couples only to the positive frequency components of the initial state in the branch wave functions (4.1). The branch wave functions in turn will be negative frequency at the endpoints of the paths on $\sigma_{0}$.

\section{Semiclassical Class Operators}

Now consider the class operators $\left\langle q^{\prime \prime}\left\|C_{h}\right\| q^{\prime}\right\rangle$ corresponding to the semiclassical coarsegrainings discussed in Section VB. For the simplest partition of the fine-grained paths into the class $c_{c l}$ of those which behave classically according to (5.3), and those which do not, $c_{q m}$, it is clear from the preceeding section that since the leading contribution to (5.5) comes from the classical path connecting $q^{\prime}$ to $q^{\prime \prime}$, to leading order in the stationary phase approximation

$$
\begin{aligned}
\left\langle q^{\prime \prime}\left\|C_{c l}\right\| q^{\prime}\right\rangle & \approx \Delta\left(q^{\prime \prime}, q^{\prime}\right) e^{i S_{c l}\left(q^{\prime \prime}, q^{\prime}\right)} \\
& \approx\left\langle q^{\prime \prime}\left\|C_{u}\right\| q^{\prime}\right\rangle
\end{aligned}
$$

while

$$
\left\langle q^{\prime \prime}\left\|C_{q m}\right\| q^{\prime}\right\rangle \approx 0 \text {. }
$$

(The zero on the right hand side of (5.15) and all such subsequent formulæ need only be true in the sense of a distribution, e.g. in the case in which the propagator is such a rapidly oscillating function that the overlap integral in (4.1) is 0 for any initial state.)

For the more refined partition defined in Section VB of $c_{c l}$ into sub-classes $c_{i j}$ of semiclassical paths according to ranges $\left\{\Delta_{i}^{\prime}, \Delta_{j}^{\prime \prime}\right\} \leftrightarrow\left\{\Delta_{i}^{\prime}, \Gamma_{j}^{\prime}\right\}$ of initial and final positions on $\sigma_{0}$ or the corresponding initial positions and momenta,

$$
\left\langle q^{\prime \prime}\left\|C_{i j}\right\| q^{\prime}\right\rangle \approx \begin{cases}\left\langle q^{\prime \prime}\left\|C_{c l}\right\| q^{\prime}\right\rangle & q^{\prime} \in \Delta_{i}^{\prime}, q^{\prime \prime} \in T_{i j} \\ 0 & \text { else }\end{cases}
$$

Here $T_{i j}$ is the region of support in $q^{\prime \prime}$ of the class operator $\left\langle q^{\prime \prime}\left\|C_{c l}\right\| q^{\prime}\right\rangle$, as defined following (5.5). (See Figure 4.)

${ }^{23}$ More generally, recall that in Section III A 1 we chose "expanding" initial and "contracting" final boundary conditions for all paths, not just the classical ones, i.e. we required $\dot{\alpha}(0)>0$ and $\dot{\alpha}(1)<0$ for all paths. 
These semiclassical results for the class operators are useful only to the extent that the stationary phase approximation can be trusted. Some techniques for evaluating restricted functional integrals such as (5.5) are discussed in [1]. Further strategies for more detailed calculation of such integrals in the context of ordinary quantum mechanics are under investigation [58].

\section{E. Semiclassical Branch Wave Functions}

Given the semiclassical class operators (5.14 - 5.15), the corresponding branch wave functions (4.1) can be evaluated as follows. Consider an initial state $\Psi\left(q^{\prime}\right)$. Then

$$
\begin{aligned}
\Psi_{c l}\left(q^{\prime \prime}\right) & =\left\langle q^{\prime \prime} \| C_{c l} \mid \Psi\right\rangle \\
& =\left\langle q^{\prime \prime}\left\|C_{c l}\right\| q^{\prime}\right\rangle \circ \Psi\left(q^{\prime}\right) \\
& \approx\left\langle q^{\prime \prime} \| C_{u} \mid \Psi\right\rangle
\end{aligned}
$$

i.e. $\Psi_{c l}\left(q^{\prime \prime}\right)$ is approximately simply the initial state propagated forward by the full propagator, while

$$
\Psi_{q m}\left(q^{\prime \prime}\right) \approx 0 .
$$

Note that (5.17-5.18) are independent of any particular properties of the initial state $\Psi\left(q^{\prime}\right)$, and are wholly a consequence of $(5.14-5.15)$.

Now consider the more refined coarse-graining of $c_{c l}$ into sub-classes $c_{i j}$ of approximately classical paths. Denote the regions of position and momentum support of $\Psi\left(q^{\prime}\right)$ on $\sigma_{0}$ by $\varepsilon$ and $\gamma$. Then

$$
\begin{aligned}
& \Psi_{i j}\left(q^{\prime \prime}\right)=\left\langle q^{\prime \prime}\left\|C_{i j}\right\| q^{\prime}\right\rangle \circ \Psi\left(q^{\prime}\right) \\
& \approx \begin{cases} & \epsilon \cap \Delta_{i}^{\prime} \neq \emptyset, \\
\left\langle q^{\prime \prime} \| C_{c l} \mid \Psi\right\rangle & \gamma \cap \Gamma_{j}^{\prime} \neq \emptyset, \\
& q^{\prime \prime} \in T_{i j} \\
0 & \text { else }\end{cases}
\end{aligned}
$$

with

$$
\Psi_{c l}\left(q^{\prime \prime}\right)=\sum_{i j} \Psi_{i j}\left(q^{\prime \prime}\right)
$$

Note that for this class of coarse-grainings, given (5.17) and (5.19) we may expect the branch wave functions to approximately satisfy the constraint (3.5), even though the coarsegraining does restrict the paths on the initial surface (see Section III A 3.) Indeed, according to $(5.19 \mathrm{~b}), \Psi_{i j}\left(q^{\prime \prime}\right)$ is just the initial data $\left\{\Delta_{i}^{\prime}, \Gamma_{j}^{\prime}\right\}$ selected by the coarse-graining and weighted by the initial state $\Psi\left(q^{\prime}\right)$, propagated by the Wheeler-DeWitt propagator (5.14). As a function on minisuperspace it is therefore concentrated around the classical trajectories connecting $\Delta_{i}^{\prime}$ to $\Delta_{j}^{\prime \prime}$. (See Figure 4.) On $\sigma_{0}$, when $q^{\prime \prime} \in \Delta_{i}^{\prime} \Psi_{i j}\left(q^{\prime \prime}\right)$ is essentially the part of the initial condition with momenta in $\Gamma_{j}^{\prime}$. In other words,

$$
\Psi_{i j}\left(q^{\prime \prime}\right) \approx \int_{\Gamma_{j}^{\prime}} d^{n} p \tilde{\Psi}^{+}(\vec{p}) \Phi_{\vec{p}}^{+}\left(q^{\prime \prime}\right)
$$

on $\sigma_{0}$ when $q^{\prime \prime} \in \Delta_{i}^{\prime}$, and is approximately zero otherwise. The restriction to positive frequency is a consequence of the fact - see Section $\mathrm{VC}$ - that the semiclassical propagator 
(5.8) couples only to the positive frequency part of the initial condition. Similarly, the semiclassical propagator is negative frequency in $\alpha^{\prime \prime}$ at the endpoints of the paths on $\sigma_{0}$, so that $\Psi_{i j}\left(q^{\prime \prime}\right)$ is negative frequency when $q^{\prime \prime} \in \Delta_{j}^{\prime \prime}$. We will put these observations to use in the next section.

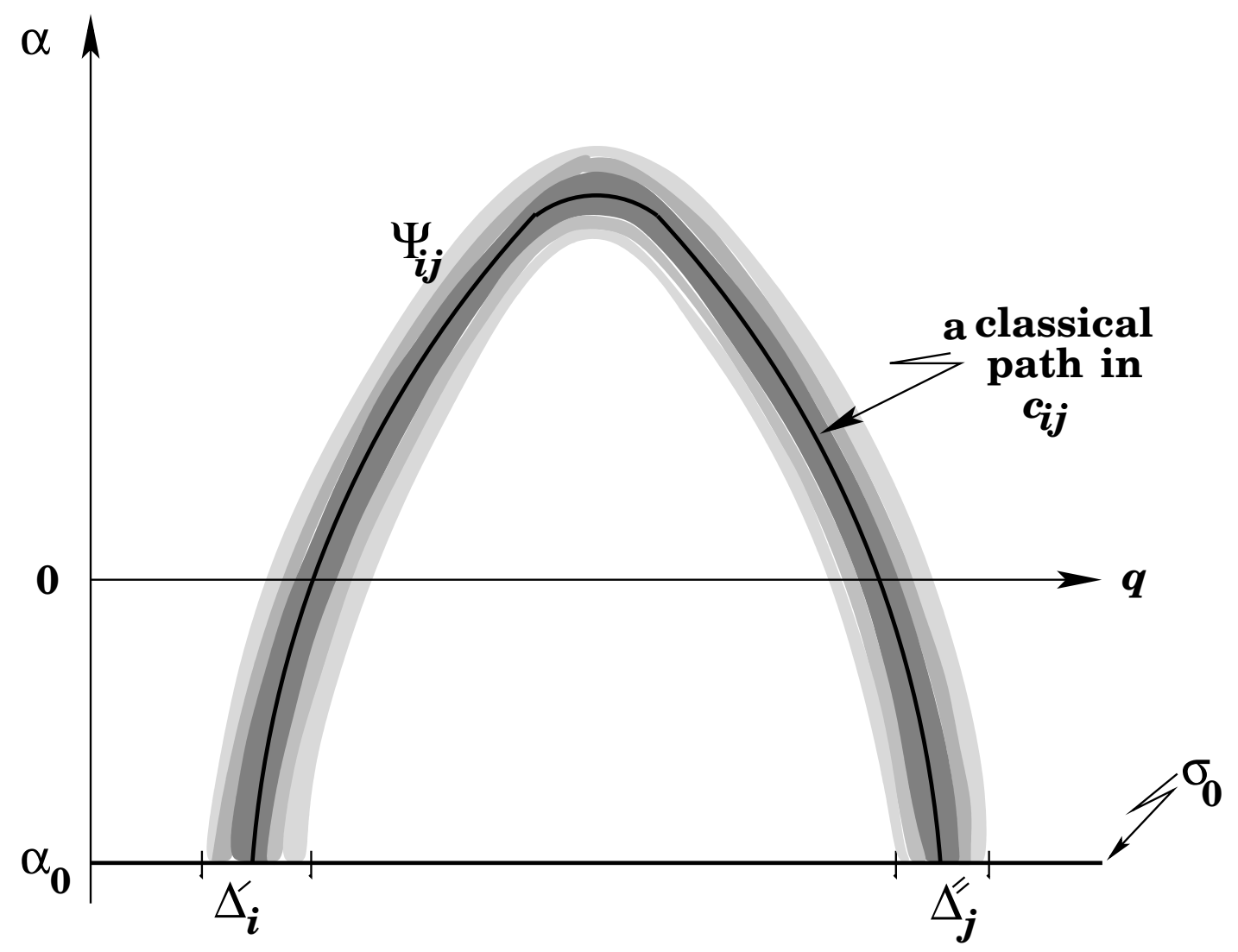

FIG. 4: Schematic representation of the region of support of the semiclassical branch wave function $\Psi_{i j}\left(q^{\prime \prime}\right)$ corresponding to the class of paths $c_{i j} . \Psi_{i j}\left(q^{\prime \prime}\right)$ is concentrated around the classical trajectories connecting $\Delta_{i}^{\prime}$ to $\Delta_{j}^{\prime \prime}$, a representative of which is plotted in the figure. The contributions of each path are weighted by the value of the initial condition $\Psi\left(q^{\prime}\right)$ in $\Delta_{i}^{\prime}$. $\Psi_{i j}\left(q^{\prime \prime}\right)$ is positive frequency in $\Delta_{i}^{\prime}$ and negative frequency in $\Delta_{j}^{\prime \prime}$. Assuming $\Psi\left(q^{\prime}\right)$ is not zero in $\Delta_{i}^{\prime}$, the shaded region will be roughly the same as $T_{i j}$, the region of support of the class operator (5.16) on minisuperspace.

\section{F. Evaluation of the Decoherence Functional: Approximately Classical Behaviour}

Given the branch wave functions (5.17-5.19), evaluation of the decoherence functional (4.22) for the partitions into approximately classical vs. highly quantum paths we have considered is straightforward.

For the coarsest-graining of paths into the classes $c_{c l}$-paths which are close to some classical path - or the remainder $c_{q m}$, decoherence is automatic because there is only one 
non-trivial branch wave function (5.17). Hence

$$
\begin{aligned}
p_{c l} & =D(c l, c l) \\
& \approx 1,
\end{aligned}
$$

i.e. the universe is predicted to approximately follow some classical trajectory - though we cannot say which one without a more refined coarse-graining - while

$$
\begin{aligned}
p_{q m} & =D(q m, q m) \\
& \approx 0
\end{aligned}
$$

independent of the choice of initial state on account of (5.15). ${ }^{24}$

To ask the question of which trajectory the universe follows for a given choice of initial state requires the more refined partition of $c_{c l}$ into the classes $c_{i j}$. Because the branch wave functions, and hence the decoherence functional (4.22), depend in this case more sensitively on the choice of initial state, we will discuss the examples of a localized initial state $\Psi_{0}$ and an initial state of WKB form $\Psi^{W K B}$ in their turn.

\section{Initial State of Localized Form}

A localized choice of initial state such as (4.25), peaked about a particular set of classical initial conditions $\left(\vec{q}_{0}, \vec{p}_{0}\right)$, leads to a decoherence functional which makes the semiclassical prediction that the universe (approximately) follows the classical path determined by the initial conditions $\left(\vec{q}_{0}, \vec{p}_{0}\right)$ as follows. In general, for a localized wave packet $\Psi^{W P}\left(q^{\prime}\right)$, define as above the regions of $\Psi^{W P}$ 's position and momentum support on $\sigma_{0}$ by $\varepsilon$ and $\gamma$. Take $\vec{q}_{0} \in \Delta_{I}^{\prime}$ and $\vec{p}_{0} \in \Gamma_{J}^{\prime}$ for some $(I, J)$, and assume for simplicity that $\varepsilon$ is largely contained in $\Delta_{I}^{\prime}$ and $\gamma$ is primarily concentrated in $\Gamma_{J}^{\prime}$. (Because of the uncertainty principle, $\varepsilon$ and $\gamma$ cannot both be compact.) In this case, through (5.19) there will then be only one nontrivial branch wave function $\Psi_{I J}^{W P}\left(q^{\prime \prime}\right)$ and decoherence will again be automatic, with the model universe consequently predicted with high probability to behave approximately like the classical trajectories in the class $c_{I J}$.

${ }^{24}$ While this would seem to be a strong statement, it is more a reflection (via (5.14-5.15)) of the fact that path integrals are dominated by paths close to the classical path than it is a claim about the actual classicality of the universe's behaviour. One would hardly expect a universe to behave in an approximately classical fashion under all circumstances or for all choices of initial state. Indeed, whether a state behaves in a recognizably classical fashion is in general as much a property of the initial state as it is of the characteristics of the restricted propagator. In this regard it is thus wise not to over-interpret the physical significance of the prediction as it stands, in that initial states normally thought of as highly non-classical such as e.g. "Schrödinger's Cat" states - superpositions of distinct localized wave packets - lead to a single non-trivial branch wave function $\Psi_{c l}$ just like any other initial state ( $c f .(5.20)$.) To detect the classically unusual features of such states requires a more refined coarse-graining such as the partition of $c_{c l}$ into the classes $c_{i j}$. A fuller understanding of this critical issue requires a more careful evaluation of the coarse-grained class operators and corresponding branch wave functions than we can give here [54]. 
To see explicitly how this comes about, consider

$$
\begin{aligned}
\Psi_{c l}^{W P}\left(q^{\prime \prime}\right) & \equiv\left\langle q^{\prime \prime} \| C_{c l} \mid \Psi^{W P}\right\rangle \\
& \equiv\left\langle q^{\prime \prime}\left\|C_{c l}\right\| q^{\prime}\right\rangle \circ \Psi^{W P}\left(q^{\prime}\right) \\
& \approx i \int_{\sigma_{0}} d^{3} q^{\prime} \Delta\left(q^{\prime \prime}, q^{\prime}\right) e^{i S_{c l}\left(q^{\prime \prime}, q^{\prime}\right)} \frac{\stackrel{\leftrightarrow}{\partial}}{\partial \alpha^{\prime}} \Psi^{W P}\left(q^{\prime}\right)
\end{aligned}
$$

For $\Psi^{W P}(q)$ localized around $\vec{q}_{0}$, it is clear that only paths with initial points $\vec{q}^{\prime}$ near $\vec{q}_{0}$ contribute significantly. Similarly, inserting (4.25) and dropping a term containing $\partial \Delta\left(q^{\prime \prime}, q^{\prime}\right) / \partial \alpha^{\prime}$

$$
\Psi_{c l}^{W P}\left(q^{\prime \prime}\right) \approx \int_{\sigma_{0}} d^{3} q^{\prime} \int \frac{d^{3} p}{\sqrt{(2 \pi)^{3}}} \frac{1}{\sqrt{2 \omega_{p}}}\left\{\omega_{p}+\frac{\partial S_{c l}\left(q^{\prime \prime}, q^{\prime}\right)}{\partial \alpha^{\prime}}\right\} \Delta\left(q^{\prime \prime}, q^{\prime}\right) e^{i S_{c l}\left(q^{\prime \prime}, q^{\prime}\right)} e^{i \vec{p} \cdot\left(\vec{q}^{\prime}-\vec{q}_{0}\right)} \tilde{\Psi}^{W P+}(\vec{p}) .
$$

Again neglecting the $q^{\prime}$ dependence of $\Delta\left(q^{\prime \prime}, q^{\prime}\right)$ as logarithmically suppressed, in the stationary phase approximation the dominant contribution to the $\vec{q}^{\prime}$ integral comes when

$$
\vec{p} \approx-\left.\frac{\partial S_{c l}\left(q^{\prime \prime}, q^{\prime}\right)}{\partial \vec{q}^{\prime}}\right|_{q^{\prime}=q_{0}}
$$

The right hand side of this equation is the initial momentum of the classical path connecting $q^{\prime}$ to $q^{\prime \prime}{ }^{25}$ Since our initial wave functions only have support on regions of $\sigma_{0}$ in which $V \approx 0$, the Hamilton Jacobi equation for $S_{c l}$ implies that $\left(\partial S_{c l} / \partial \vec{q}\right)^{2}=\left(\partial S_{c l} / \partial \alpha\right)^{2}$. From (5.13), $\partial S_{c l} / \partial \alpha^{\prime}$ is positive, so that when (5.26) holds, $\omega_{p}=p \approx \partial S_{c l} / \partial \alpha^{\prime}$, and thus

$$
\Psi_{c l}^{W P}\left(q^{\prime \prime}\right) \approx \int \frac{d^{3} p}{\sqrt{(2 \pi)^{3}}} \sqrt{2 \omega_{p}} \tilde{\Psi}^{W P+}(\vec{p}) \int_{\sigma_{0}} d^{3} q^{\prime} \Delta\left(q^{\prime \prime}, q^{\prime}\right) e^{i S_{c l}\left(q^{\prime \prime}, q^{\prime}\right)} e^{i \vec{p} \cdot\left(\vec{q}^{\prime}-\vec{q}_{0}\right)} .
$$

Performing the integration over $\vec{p}$, we therefore see that the branch wave function is suppressed when $\tilde{\Psi}^{W P}(\vec{p})$ is small in the neighborhood of the initial momentum of the classical path connecting $q_{0}$ to $q^{\prime \prime}$. The choice of initial condition $\Psi^{W P}\left(q^{\prime}\right)$ on $\sigma_{0}$, therefore, singles out the classical path specified by $\left(\vec{q}_{0}, \vec{p}_{0}\right): \Psi_{c l}^{W P}\left(q^{\prime \prime}\right)$ is peaked around the classical path preferred by the initial condition. Through (5.19) it is then clear that the only nontrivial branch wave function for this coarse-graining is the $\Psi_{I J}^{W P}\left(q^{\prime \prime}\right)$ corresponding to the class $c_{I J}$ which contains the classical path determined by the initial conditions $\left(\vec{q}_{0}, \vec{p}_{0}\right)$ :

$$
\Psi_{i j}^{W P}\left(q^{\prime \prime}\right) \approx \begin{cases}\Psi_{c l}^{W P}\left(q^{\prime \prime}\right) & i=I, j=J \\ 0 & \text { else }\end{cases}
$$

This is merely a more refined statement of (5.2).

${ }^{25}$ Note that the specification of $\dot{\alpha}(0)$ is implicit. To see this, note that $\partial S_{c l} / \partial \alpha$ is not independent of $\partial S_{c l} / \partial \vec{q}$ : these quantities are related by the Hamilton-Jacobi equation $\nabla_{A} S \nabla^{A} S+V=0$ for $S_{c l}-$ effectively the constraint (4.24) with $p_{A}=\dot{q}_{A} / 2 N=-\nabla_{A} S$. Therefore when (5.26) holds, $\dot{\alpha}$ is given by $\partial S / \partial \alpha$ through (5.13); $N$ is determined by demanding consistency of the solution with the constraint ( $c f$. the variant of this argument we give below while discussing the case of an initial state of WKB form.) 
The situation for the WKB choice of initial state (4.26) is a little different, though familiar from conventional treatments of quantum cosmology.

Let us evaluate the classical branch wave function corresponding to an initial state of the WKB form (4.26).

$$
\begin{aligned}
\Psi_{c l}^{W K B}\left(q^{\prime \prime}\right) & =\left\langle q^{\prime \prime}\left\|C_{c l}\right\| q^{\prime}\right\rangle \circ \Psi^{W K B}\left(q^{\prime}\right) \\
& =i \int_{\sigma_{0}} d \Sigma^{\prime A}\left\{A\left(q^{\prime}\right)\left[\left\langle q^{\prime \prime}\left\|C_{c l}\right\| q^{\prime}\right\rangle \stackrel{\leftrightarrow}{A}_{A}^{\prime} e^{i W\left(q^{\prime}\right)}\right]-\left\langle q^{\prime \prime}\left\|C_{h}\right\| q^{\prime}\right\rangle e^{i W\left(q^{\prime}\right)} \nabla_{A}^{\prime} A\left(q^{\prime}\right)\right\} \\
& \approx i \int_{\sigma_{0}} d \Sigma^{\prime A} \Delta\left(q^{\prime \prime}, q^{\prime}\right) e^{i S_{c l}\left(q^{\prime \prime}, q^{\prime}\right)} A\left(q^{\prime}\right) e^{i W\left(q^{\prime}\right)}\left[\nabla_{A}^{\prime} W\left(q^{\prime}\right)-\nabla_{A}^{\prime} S_{c l}\left(q^{\prime \prime}, q^{\prime}\right)\right]
\end{aligned}
$$

In $(5.29 \mathrm{c})$ we have dropped the terms containing gradients of the more slowly varying terms $A\left(q^{\prime}\right)$ and $\Delta\left(q^{\prime \prime}, q^{\prime}\right)$. In this form, it is clear that the dominant contribution to the integral comes when the stationary phase condition

$$
\nabla_{A}^{\prime} W\left(q^{\prime}\right)=-\nabla_{A}^{\prime} S_{c l}\left(q^{\prime \prime}, q^{\prime}\right)
$$

is satisfied. The result is thus

$$
\Psi_{c l}^{W K B}\left(q^{\prime \prime}\right) \approx 2 i \int_{\sigma_{0}} d \Sigma^{\prime A} \Delta\left(q^{\prime \prime}, q^{\prime}\right) A\left(q^{\prime}\right) \nabla_{A}^{\prime} W\left(q^{\prime}\right) e^{i S_{c l}\left(q^{\prime \prime}, q^{\prime}\right)} e^{i W\left(q^{\prime}\right)} .
$$

The quantity on the right hand side of (5.30) is just the initial momentum of the classical path connecting $q^{\prime}$ to $q^{\prime \prime}$. Note that it is a statement about the complete minisuperspace gradient of $S_{c l}$, not merely its projection into the $\alpha=$ constant surface $\sigma_{0}$. We are free to assert that contributions to the integral (5.29c) are suppressed when (5.30) does not hold because of the independence of the branch wave functions on the choice of initial surface: wiggling $\sigma_{0}$ around a bit in the neighborhood of $q^{\prime}$, yet keeping $q^{\prime}$ fixed on $\sigma_{0}$, yields a family of stationarity conditions for the dominant contribution to the branch wave functions. For them all to be true, (5.30) must hold. Alternatively, note that $\Psi^{W K B}(q)$ is a solution to the constraint (3.5). Dropping gradients of $A(q)$ at lowest order, this means that $W(q)$ obeys the same Hamilton-Jacobi equation as does $S_{c l}\left(q^{\prime \prime}, q^{\prime}\right)$. Thus, with "expanding" initial conditions on the paths, this means that the "time" component of (5.30) holds whenever the "spatial" part does, so that (5.30) follows from its projection into $\sigma_{0}$.

The significance of (5.30) is that $\Psi_{c l}^{W K B}\left(q^{\prime \prime}\right)$ is peaked around the family of classical trajectories emanating from points $q_{0}^{\prime}$ on $\sigma_{0}$ which have initial momentum $\left.\nabla_{A}^{\prime} W\left(q^{\prime}\right)\right|_{q^{\prime}=q_{0}^{\prime}}$, with a relative weight controlled by the WKB prefactor $A\left(q^{\prime}\right)$. This is simply because $\Psi_{c l}^{W K B}\left(q^{\prime \prime}\right)$ is suppressed when the classical path from $q^{\prime}$ to $q^{\prime \prime}$ does not have initial momentum given by (5.30). When the classical trajectory from $q^{\prime}$ to $q^{\prime \prime}$ has momentum $\nabla_{A}^{\prime} W\left(q^{\prime}\right)$, but $A\left(q^{\prime}\right)$ is small, the integral is similarly suppressed.

Now let us consider the situation of the more refined coarse-graining $\left(c_{q m}, c_{i j}\right)$, where the classes $c_{i j}$ defined in Section VB are the partitions of semiclassical paths according to ranges $\left\{\Delta_{i}^{\prime}, \Delta_{j}^{\prime \prime}\right\} \leftrightarrow\left\{\Delta_{i}^{\prime}, \Gamma_{j}^{\prime}\right\}$ of initial and final positions on $\sigma_{0}$ or the corresponding initial positions and momenta. Clearly, only the classes $c_{i j}$ containing paths with initial points $q^{\prime}$ in regions where $A\left(q^{\prime}\right)$ is not small can have non-negligible branch wave functions. Of these, the classes containing the classical path emanating from $q^{\prime}$ with initial momentum $\nabla_{A}^{\prime} W\left(q^{\prime}\right)$ 
will possess the dominant branch wave functions; the relative magnitude of these branch wave functions will be controlled by the WKB amplitude $A(q)$. These wave functions will as before be concentrated around the corresponding classical trajectories, and are given as in $(5.19)$ by

$$
\Psi_{i j}^{W K B}\left(q^{\prime \prime}\right) \approx \begin{cases} & \epsilon \cap \Delta_{i}^{\prime} \neq \emptyset \\ \Psi_{c l}^{W K B}\left(q^{\prime \prime}\right) & \gamma \cap \Gamma_{j}^{\prime} \neq \emptyset \\ & q^{\prime \prime} \in T_{i j} \\ 0 & \text { else }\end{cases}
$$

For these more refined coarse-grainings, therefore, we have a collection of non-trivial branch wave functions, and the decoherence functional has more to tell us. It is in particular of interest to ask whether the coarse-graining $\left(c_{q m}, c_{i j}\right)$ decoheres. For this purpose it suffices to consider the non-negligible branch wave functions and examine their overlap:

$$
\begin{aligned}
D\left(c_{i j}, c_{k l}\right) & =\mathcal{N} \Psi_{k l}^{W K B} \bullet \Psi_{i j}^{W K B} \\
& =\mathcal{N}\left\langle q^{\prime \prime} \| C_{k l} \mid \Psi^{W K B}\right\rangle \bullet\left\langle q^{\prime \prime} \| C_{i j} \mid \Psi^{W K B}\right\rangle
\end{aligned}
$$

The integrals in (5.33b) are to be evaluated on $\sigma_{0}$. According to $(5.32), \Psi_{i j}^{W K B}\left(q^{\prime \prime}\right)$ is peaked around the classical paths in the class $c_{i j}$. As at least one pair of the initial or final intervals $\Delta^{\prime}$ or $\Delta^{\prime \prime}$ will be disjoint on $\sigma_{0}$ (see Figure 4) - and even if one of the initial or final intervals coincide, the momentum support will be disjoint - we see that the overlap integral in (5.33) will be small, and so we have approximate decoherence of the coarse-graining $\left(c_{q m}, c_{i j}\right)$ :

$$
D(q m, i j) \approx 0
$$

In other words, the WKB branch wave functions approximately decohere because they are approximately macroscopically distinct. A higher degree of decoherence would require correlations with additional degrees of freedom.

We now estimate the relative probabilities $p_{i j}=D\left(c_{i j}, c_{i j}\right)$ of the various members of the family of classical paths preferred by the WKB initial condition. The answer will be a precise version of the familiar heuristic rule of quantum cosmology according to which these probabilities are given by fluxes throuh a spacelike surface in minisuperspace. (See [28, 44] for related results.)

The branch wave functions $\Psi_{i j}^{W K B}\left(q^{\prime \prime}\right)$ have support on $\sigma_{0}$ essentially only in $\Delta_{i}^{\prime}$ and $\Delta_{j}^{\prime \prime}$. Recall that in Section IV F we chose our initial state $\Psi^{W K B}\left(q^{\prime}\right)$ to be positive frequency in $\alpha^{\prime}$, and that, as noted at the end of Section VE, $\Psi_{i j}^{W K B}\left(q^{\prime \prime}\right)$ is negative frequency at the ends of the paths on $\sigma_{0} \cdot \Psi_{i j}^{W K B}\left(q^{\prime \prime}\right)$ is therefore positive frequency in $\Delta_{i}^{\prime}$ and negative frequency in $\Delta_{j}^{\prime \prime}$. Further, because the current $J_{A}=-(i / 2) \Psi^{*} \stackrel{\leftrightarrow}{\nabla}_{A} \Psi$ is conserved and $\Psi_{i j}^{W K B}\left(q^{\prime \prime}\right)$ vanishes on any spacelike surface $\sigma_{f}$ far to the future of $T_{i j}$ (i.e. at very large values of $\alpha$ - see Figure 4 ), integrating $\nabla_{A} J^{A}=0$ over a volume bounded by $\sigma_{0}$ and $\sigma_{f}$ we see that

$$
\begin{aligned}
\int_{\sigma_{0}} d \Sigma^{A} J_{A} & =\int_{\Delta_{i}^{\prime}} d \Sigma^{A} J_{A}+\int_{\Delta_{j}^{\prime \prime}} d \Sigma^{A} J_{A} \\
& =0
\end{aligned}
$$

from which we learn that

$$
\Psi_{i j}^{W K B+} \circ \Psi_{i j}^{W K B+}+\Psi_{i j}^{W K B-} \circ \Psi_{i j}^{W K B-}=0
$$


on $\sigma_{0}$. Thus

$$
\begin{aligned}
p_{i j} & =\mathcal{N} \Psi_{i j}^{W K B} \bullet \Psi_{i j}^{W K B} \\
& =\mathcal{N}\left(\Psi_{i j}^{W K B+} \circ \Psi_{i j}^{W K B+}-\Psi_{i j}^{W K B-} \circ \Psi_{i j}^{W K B-}\right) \\
& =2 \mathcal{N} \Psi_{i j}^{W K B+} \circ \Psi_{i j}^{W K B+} \\
& \approx 2 \mathcal{N} i \int_{\Delta_{i}^{\prime}} d \Sigma^{\prime A} \Psi_{i j}^{W K B *}\left(q^{\prime}\right) \stackrel{\leftrightarrow}{\nabla}_{A} \Psi_{i j}^{W K B}\left(q^{\prime}\right) .
\end{aligned}
$$

In (5.37d) the most obvious vestige of the effect of the class operator $C_{i j}$ is the restriction, via (5.32), of the domain of integration on $\sigma_{0}$ to $\Delta_{i}^{\prime}$, though the conditions on the final position $\Delta_{j}^{\prime \prime}$ (equivalently, initial momentum $\Gamma_{j}^{\prime}$ ) corresponding to the class $c_{i j}$ are still present through (5.32) - cf. (5.21) - and the connection (5.30).

Inserting the semiclassical form (4.26), dropping gradients of the slowly varying factor $A$, and evaluating the remaining integral at the center $q_{i}^{\prime}=\left(\alpha_{0}, \vec{q}_{i}^{\prime}\right)$ of the domain of integration $\Delta_{i}^{\prime}$ on $\sigma_{0}$,

$$
\begin{aligned}
p_{i j} & \approx 2 \mathcal{N} i \int_{\Delta_{i}^{\prime}} d \Sigma^{\prime A}|A|^{2} 2 i \nabla_{A} W \\
& \approx-\mathcal{N}\left|A\left(q_{i}^{\prime}\right)\right|^{2} \nabla_{A}^{\prime} W\left(q_{i}^{\prime}\right) d \Sigma_{i}^{\prime A}
\end{aligned}
$$

when the projection of $\nabla_{A}^{\prime} W\left(q^{\prime}\right)$ into $\sigma_{0}$ is in $\Gamma_{j}^{\prime}$, and is zero otherwise. (A factor of 4 has been absorbed into the normalization factor $\mathcal{N}$ in the second line.) If $\sigma_{0}$ is chosen to be a surface of constant $\alpha=\alpha_{0}$ and recalling again that the initial state was chosen to be "positive frequency" so that $\partial W / \partial \alpha^{\prime}<0,(5.38 \mathrm{~b})$ reduces to

$$
p_{i j} \approx \mathcal{N}\left|A\left(q_{i}^{\prime}\right)\right|^{2}\left|\frac{\partial W}{\partial \alpha^{\prime}}\left(q_{i}^{\prime}\right)\right| \Delta_{i}^{\prime}
$$

so long as $\partial W / \partial \vec{q}^{\prime}\left(q_{i}^{\prime}\right) \in \Gamma_{j}^{\prime}$. The factor $\mathcal{N}$ can be calculated in this approximation by the requirement that the probabilities sum to unity.

Further, it may be worth noting that in arriving at (5.37d) the only properties of the branch wave functions we employed were that the wave functions (i) vanish as $\alpha \rightarrow \infty$; (ii) are positive frequency in $\Delta_{i}^{\prime}$; and (iii) negative frequency in $\Delta_{j}^{\prime \prime}$. These properties derive essentially from the corresponding properties of the propagator, which in turn follow from our boundary conditions on the paths - namely, that they begin and end on $\sigma_{0}$ with "expanding" initial and "contracting" final conditions - and did not depend on any specific property of the WKB form of the initial state. Thus, for this class of coarse-grainings we can write more generally

$$
\begin{aligned}
p_{i j} & \approx-4 \mathcal{N} \int_{\Delta_{i}^{\prime}} d \Sigma^{A} J_{i j A}^{+} \\
& \approx-\mathcal{N} J_{i j A}^{+}\left(q_{i}^{\prime}\right) d \Sigma_{i}^{\prime A}
\end{aligned}
$$

on $\sigma_{0}$, where $J_{i j A}^{+}$is the current constructed from the positive frequency part of the branch wave function $\Psi_{i j}$ for any initial state. (A factor of 4 has again been absorbed into $\mathcal{N}$ in the second line.)

We have thus recovered a version appropriate to the present formalism of the familiar $[1,6,59]$ " $J \cdot d \Sigma$ " rule of quantum cosmology according to which $J_{A} d \Sigma^{A}\left(\approx|A|^{2} \nabla_{A} W d \Sigma^{A}\right.$ 
for WKB initial states) gives the relative probabilities of the classical trajectories passing through elements $d \Sigma^{A}$ of spacelike surfaces in minusperspace. In our formalism, however, that rule need only be applied on the initial surface $\sigma_{0}$, and can be applied to initial states more general than just those of WKB form.

\section{CONCLUSION}

We have constructed a sum-over-histories generalized quantum theory for the Bianchi IX cosmologies. This fully four-dimensional formalism allows predictions to be made concerning sets of (reparametrization invariant) alternative histories of such a universe. In particular, the predictions of the theory are not restricted to those defined only at a moment of "time", as is typical of canonical theories, and hence are not restricted to alternatives defined only by observables on superspace. Many questions of physical interest are not expressible in terms of alternatives "at one moment of time"; the generality of the alternatives about which it is possible to make predictions, and the naturalness with which the formalism accommodates them, are notable conceptual advantages of this quantization scheme.

Partitions of histories of physical interest include partitions according to whether or not the universe becomes singular (by, for instance, the standard discussed in Section III); partitions by ranges of values of the volume and degree of anisotropy $\beta_{ \pm}$the universe has achieved a given proper time after the initial condition; and partitions into classes of those histories which are close to some classical cosmological trajectory, and those which are not. Approximate classicality, in the sense of obeying the classical equations of motion, is exemplary of a prediction concerning an alternative that is defined over time, not just at a single moment. For this last set of alternatives, we showed in Section V that, for indifferent final conditions and particular choices of initial condition, the generalized quantum theory predicts with a probability near one that the universe behaves semiclassically, approximately following the classical trajector(ies) preferred by the initial condition. This is a sensible prediction, and stands in contrast to the less intuitive predictions of some other quantizations of Bianchi IX (as mentioned at the beginning of Section V.)

There is much that remains to be be done with this formulation of a quantum mechanics for cosmology. The most pressing issue concerns the development of techniques for more careful evaluation of the class operators (3.14) for various choices of coarse-graining, and a correspondingly improved understanding of the limitations of the approximations employed in the present work. Application of the general formalism described here to a detailed analysis of specific examples of analytic or numerical classical solutions will then be of interest.

Other issues include the extension of the definition of final boundary conditions which are "indifferent" to the paths to the case where the universe expands forever, thereby enabling consideration of ever-expanding models and the calculation of the probability that a Bianchi IX universe with cosmological constant will "tunnel through" to a state of eternal expansion. (Once the technical issues are sorted out, defining the decoherence functional through (4.22) using the Rieffel induced innner product is likely to be a constructive approach to this problem.) Predictions relevant to modern day cosmological observations and questions of environmental decoherence will require the inclusion of inhomogeneous modes of matter fields and high frequency (gravity wave or graviton) modes of the gravitational field.

In all cases, the rules for performing the required calculations are clearly defined: specify the boundary conditions; set out the partition defining the physical alternatives it is desired 
to study; compute the branch wave functions (4.1) corresponding to those partitions and initial conditions, and employ the decoherence functional (4.22) to determine whether this set of alternatives is consistent. If it is, the diagonal elements of the decoherence functional inform us of the probabilities for those alternatives according to (3.1).

Finally, it is perhaps worth emphasizing that the essence of the predictive framework we have here described should be straightforward to generalize to quantum-gravitational technologies - string theory or loop quantum gravity, for example - other than the particular path-integral formulation we have offered. (The essential technical step lies in the definition of the restricted propagators (3.4).)

\section{Acknowledgments}

We would like to thank Don Marolf and John Whelan for useful conversations, and Thea Howard for creating the figures. This work was supported in part by NSF Grants PHY-90-08502, PHY-95-07065, and PHY-02-44764. D.C. was supported in part also by the Canadian Institute for Theoretical Astrophysics (CITA) and the Natural Sciences and Engineering Research Council of Canada (NSERC).

\section{APPENDIX: CLASS OPERATORS SATISFY CONSTRAINTS}

This appendix is devoted to demonstrating that the class operator matrix element $\left\langle q^{\prime \prime}\left\|C_{h}\right\| q^{\prime}\right\rangle$ defined by (3.4) satisfy an operator form of the constraints. More concretely, we show that, for the kind of coarse-grainings defining classicality discussed in Section $\mathrm{V}$,

$$
H_{q^{\prime \prime}}\left\langle q^{\prime \prime}\left\|C_{h}\right\| q^{\prime}\right\rangle=0
$$

when $q^{\prime \prime}$ is outside the region of configuration space where the paths are limited by the coarse graining and is not equal to $q^{\prime}$. We consider a coarse graining where the paths to the future of some $q^{0}=\alpha^{*}$ are constrained to lie in a tube $T$ about a particular classical path. We denote the class operator corresponding to this particular coarse graining by $C_{T}$. We will show that (A.1) holds for $\alpha<\alpha^{*}$. That can be used to show that the product (3.8) is independent of the choice of initial hypersurface $\sigma^{\prime}$ and final hypersurface $\sigma^{\prime \prime}$ provided they are both in the region $\alpha<\alpha^{*}$. There are other coarse grainings for which (A.1) holds (see, e.g. [1, Section VII].) We focus on this one because it is relevant for classicality.

It is convenient to work in $\dot{N}=0$ "gauge" of (3.13). The class operator matrix elements can then be written in the form (3.16) with $\left\langle q^{\prime \prime} N\left\|C_{T}\right\| q^{\prime} 0\right\rangle$ given by

$$
\left\langle q^{\prime \prime} N\left\|C_{T}\right\| q^{\prime} 0\right\rangle=\int \delta q_{q^{\prime}}^{q^{\prime \prime}} e_{T}\left[q^{A}(s)\right] \exp \left(i \int_{0}^{N} d s\left[\frac{1}{4} G_{A B} \frac{d q^{A}}{d s} \frac{d q^{B}}{d s}-V\right]\right) .
$$

Here the integral is over all paths connecting $q^{\prime}$ to $q^{\prime \prime}$ and $e_{T}\left[q^{A}(s)\right]$ is the characteristic functional for the tube: This is 1 for $\alpha<\alpha^{*}$ (paths unrestricted), 1 if the path $q^{A}(s)$ lies in the tube for $\alpha>\alpha^{*}$, and 0 if any part lies outside it for $\alpha>\alpha^{*}$. (A.2) is just (3.17b) written in terms of this characteristic functional.

We next show that $\left\langle q^{\prime \prime} N\left\|C_{T}\right\| q^{\prime} 0\right\rangle$ obeys the "Schrödinger equation"

$$
i \frac{\partial}{\partial N}\left\langle q^{\prime \prime} N\left\|C_{T}\right\| q^{\prime} 0\right\rangle=H_{q^{\prime \prime}}\left\langle q^{\prime \prime} N\left\|C_{T}\right\| q^{\prime} 0\right\rangle \text {. }
$$


The demonstration is the same as Feynman's original demonstration that the path integral defining the propagator in non-relativistic quantum mechanics satisfies the Schrödinger equation [60]. The only novelty is the characteristic functional $e_{T}\left[q^{A}(s)\right]$ in (A.2). We define the path integral in (A.2) as the limit of integrals over paths skeletonized on $J+1$ uniformly spaced slices of $s$ as $J$ tends to infinity. Specifically, let $s_{0}=0, s_{1}, s_{2} \cdots, s_{J}=N$ be slices spaced by $\epsilon=N / J$. Let $q_{k}^{A}$ be the value of $q^{A}$ on slice $k$. Then,

$$
\begin{aligned}
& \left\langle q^{\prime \prime} N\left\|C_{T}\right\| q^{\prime} 0\right\rangle=\lim _{J \rightarrow \infty} \int \prod_{k=1}^{J-1}\left(\prod_{A=0}^{3} d q_{k}^{A}\right)\left(\frac{-1}{4 \pi i \epsilon}\right)^{2} e_{T}\left(q_{k}^{A}\right) \\
& \times \exp \left\{i \sum_{k=1}^{J} \epsilon\left[\frac{1}{4} G_{A B}\left(\frac{q_{k}^{A}-q_{k-1}^{A}}{\epsilon}\right)\left(\frac{q_{k}^{B}-q_{k-1}^{B}}{\epsilon}\right)-V\left(q_{k}^{A}\right)\right]\right\} .
\end{aligned}
$$

Here, $e_{T}\left(q^{A}\right)$ is the characteristic function for the tube equal to 1 inside the tube and zero outside for $\alpha>\alpha^{*}$ and equal to 1 for $\alpha<\alpha^{*}$ (unrestricted). The product of these functions on different slices make up the functional $e_{T}\left[q^{A}(s)\right]$ in (A.2). The measure factor $(-1 / 4 \pi i \epsilon)^{2}$ can be deduced from the Liouville measure on phase-space $[1,61]$ recalling that $G_{A B}=\operatorname{diag}(-1,1,1,1)$.

The successive integrations in (A.4) evolve $\left\langle q^{\prime \prime} N\left\|C_{T}\right\| q^{\prime} 0\right)$ foward in $N$. Thus,

$$
\begin{aligned}
\left\langle q^{\prime \prime} N\left\|C_{T}\right\| q^{\prime} 0\right\rangle=\lim _{\epsilon \rightarrow 0} \int \prod_{A=0}^{3} d q_{J-1}^{A}\left(\frac{-1}{4 \pi i \epsilon}\right)^{\frac{1}{2}} e_{T}\left(q_{J}^{A}\right) \\
\quad \times \exp \left\{i \epsilon\left[\frac{1}{4} G_{A B}\left(\frac{q^{\prime \prime}-q_{J-1}^{A}}{\epsilon}\right)\left(\frac{q^{\prime \prime B}-q_{J-1}^{B}}{\epsilon}\right)-V\left(q_{J}^{A}\right)\right]\right\} \\
\quad \times\left\langle q_{J-1} N-\epsilon\left\|C_{T}\right\| q^{\prime} 0\right\rangle .
\end{aligned}
$$

Now restrict attention to $\alpha^{\prime \prime}<\alpha^{*}$ where $e_{T}\left(q_{J}^{A}\right)=1$. The integration over $q_{J-1}^{A}$ is restricted by the factor $e_{T}\left(q_{J-1}^{A}\right)$ in $\left\langle q_{J-1} N-\epsilon\left\|C_{T}\right\| q^{\prime} 0\right\rangle$. As $\epsilon \rightarrow 0$, an increasingly narrow range of $q_{J-1}^{A}$ near $q^{\prime \prime A}$ provides the only significant contribution to the integral. The result is therefore no different from the integral without any restriction in this limit for $\alpha^{\prime \prime}<\alpha^{*}$. As in non-relativistic quantum mechanics (A.5) implies the "Schrödinger equation" (A.3).

Integrating (A.3) from $N=0$ to $N=\infty$ gives

$$
H_{q^{\prime \prime}}\left\langle q^{\prime \prime}\left\|C_{T}\right\| q^{\prime}\right\rangle=i\left[\left\langle q^{\prime \prime} \infty\left\|C_{T}\right\| q^{\prime} 0\right\rangle-\left\langle q^{\prime \prime} 0\left\|C_{T}\right\| q^{\prime} 0\right\rangle\right]
$$

for $\alpha^{\prime \prime}<\alpha^{*}$. The first term on the right-hand side vanishes because what was a localized "wave packet" at $N=0$ has spread over all of configuration space by $N=\infty$. The second term vanishes if $q^{\prime \prime} \neq q^{A}$ for all $A$. That is because for small $N=\epsilon$

$$
\left\langle q^{\prime \prime} \epsilon\left\|C_{T}\right\| q^{\prime} 0\right\rangle=\left(\frac{-1}{4 \pi i \epsilon}\right)^{2} \exp \left[i \epsilon \frac{1}{4} G_{A B}\left(\frac{q^{\prime \prime A}-q^{\prime A}}{\epsilon}\right)\left(\frac{q^{\prime \prime B}-q^{\prime B}}{\epsilon}\right)\right]
$$

from (A.4) when $\alpha^{\prime \prime}<\alpha^{*}$. But this is a representation of a $\delta$-function as $\epsilon \rightarrow 0$, and we demonstrate (A.1) for $\alpha^{\prime \prime}<\alpha^{*}$. The right hand side of (A.1) vanishes when $q^{\prime} \neq q^{\prime \prime}$, and the class operators thus in this sense satisfy an operator form of the constraints. (More precisely, we see that these class operators are Green functions for the Wheeler-DeWitt Hamiltonian. 
For more on the question of when class operators constructed according to (3.14) satisfy constraints, see $[28,39,41,43-45]$.)

[1] J. B. Hartle, in Gravitation and Quantizations, edited by B. Julia and J. Zinn-Justin (North Holland, Amsterdam, 1995); gr-qc/9304006.

[2] C. J. Isham and N. Linden, J. Math. Phys. 355452 (1995).

[3] D. Craig, "The Geometry of Consistency: Decohering Histories in Generalized Quantum Theory", quant-ph/9704031.

[4] C. W. Misner, Phys. Rev. 1861319 (1969).

[5] M. A. H. MacCallum, in General Relativity: An Einstein Centenary Survey, edited by S. W. Hawking and W. Israel (Cambridge University Press, Cambridge, 1979).

[6] J. J. Halliwell, in Quantum Cosmology and Baby Universes: Proceedings of the 1989 Jerusalem Winter School for Theoretical Physics, edited by S. Coleman, J. B. Hartle, T. Piran, and S. Weinberg (World Scientific, Singapore, 1991).

[7] Dynamical Systems in Cosmology, edited by J. Wainwright and G. F. R. Ellis (Cambridge University Press, Cambridge, 1997).

[8] A. Ashtekar, R. Tate, and C. Ugla, Int. J. Mod. Phys. D2 15 (1993).

[9] R. M. Wald, Phys. Rev. D48 R2377 (1993).

[10] A. Higuchi and R. M. Wald, Phys. Rev. D51 544 (1995).

[11] D. Marolf, Class. Quant. Grav. 121199 (1995); ibid. 121441 (1995).

[12] J. B. Hartle, in Quantum Cosmology and Baby Universes: Proceedings of the 1989 Jerusalem Winter School for Theoretical Physics, edited by S. Coleman, J. B. Hartle, T. Piran, and S. Weinberg (World Scientific, Singapore, 1991).

[13] C. J. Isham, J. Math. Phys. 232157 (1994).

[14] R. Griffiths, J. Stat. Phys. 36219 (1984).

[15] R. Omnès, J. Stat. Phys. 53893 (1988); ibid. 53, 933 (1988); ibid. 53, 957 (1988); ibid. 57, 357 (1989); Ann. Phys. 201, 354 (1990); Rev. Mod. Phys. 64, 339 (1992); and Interpretation of Quantum Mechanics (Princeton University Press, Princeton, 1994).

[16] M. Gell-Mann and J.B. Hartle, in Complexity, Entropy, and the Physics of Information, SFI Studies in the Sciences of Complexity, Volume VIII, edited by W. Zurek (Addison-Wesley, Reading, 1990), or in Proceedings of the 3rd International Symposium on the Foundations of Quantum Mechanics in the Light of New Technology, edited by S. Kobayashi, H. Ezawa, M. Murayama, and S. Nomura (Physical Society of Japan, Tokyo, 1990).

[17] K. Kuchar, in General Relativity and Relativistic Astrophysics, Proceedings of the Fourth Canadian Conference, Winnipeg, Canada, edited by G. Kunstatter, D. Vincent, and J. Williams (World Scientific, Singapore, 1992); C. J. Isham, in Recent Aspects of Quantum Fields, edited by H. Mitter and H. Gausterer (Springer-Verlag, Berlin, 1992); and C. J. Isham, in Integrable Systems, Quantum Groups, and Quantum Field Theories, edited by L. A. Ibort and M. A. Rodriguez (Kluwer, New York, 1993).

[18] M. Ryan and L. C. Shepley, Homogeneous Relativistic Cosmologies (Princeton University Press, Princeton, 1975).

[19] M. A. H. MacCallum, in Cargèse Lectures in Physics, volume 6, edited by E. Schatzman (Gordon and Breach, New York, 1973).

[20] R. M. Wald, General Relativity (University of Chicago Press, Chicago, 1984). 
[21] C. W. Misner, Essay for Gravity Research Foundation, New Boston (1967); Ap. J. 151, 431 (1968).

[22] A. H. Taub, Ann. Math. 53472 (1951); G. F. R. Ellis and M. A. H. MacCallum, Commun. Math. Phys. 12108 (1969).

[23] L. Bianchi, Mem. Soc. It. della Sc. (dei XL)11 267 (1897); reprinted in L. Bianchi, Opere (Edizioni Cremonese, 1952-1958), vol. 9, 16.

[24] M. A. MacCallum and A. H. Taub, Commun. Math. Phys. 25173 (1972); M. P. Ryan, J. Math. Phys. 15812 (1972); G. E. Sneddon, J. Phys. A9 229 (1976); R. T. Jantzen, Commun. Math. Phys. 64211 (1979); R. T. Jantzen, Nuovo Cim. 55B 161 (1980); M. P. Ryan and S. M. Waller, gr-qc/9709012 (1984); J. M. Pons and L. C. Shepley, Phys. Rev. D58 024001 (1998).

[25] C. W. Misner, K. S. Thorne, and J. A. Wheeler, Gravitation (Freeman, San Francisco, 1973).

[26] R. M. Wald, Phys. Rev. D28 R2118 (1983).

[27] C. W. Misner, Phys. Rev. Lett. 221071 (1969).

[28] J. J. Halliwell and J. Thorwart, Phys. Rev. D64 124018 (2001).

[29] X. F. Lin and R. M. Wald, Phys. Rev. D40 3280 (1989); Phys. Rev. D41, 2444 (1990).

[30] C. Teitelboim, Phys. Rev. D25 3159 (1983); Phys. Rev. D28, 297 (1983); Phys. Rev. D28, 310 (1983); Phys. Rev. Lett. 50, 705 (1983).

[31] R. Sorkin, Int. J. Theor. Phys. 33523 (1994).

[32] G. T. Horowitz and D. Marolf, Phys. Rev. D52 5670 (1995).

[33] M. Henneaux and C. Teitelboim, Ann. Phys. 143127 (1983).

[34] K. Kuchař, private communication.

[35] H. Dowker and J. Halliwell, Phys. Rev. D46 1580 (1992).

[36] M. Gell-Mann and J.B. Hartle, Phys. Rev. D47 3345 (1993).

[37] E. Joos, H. D. Zeh, C. Kiefer, D. Giulini, J. Kupsch, and I.-O. Stamatescu, Decoherence and the Appearance of a Classical World in Quantum Theory, Second Edition (Springer-Verlag, Berlin, 2003).

[38] C. Teitelboim, Phys. Rev. D25 3159 (1983).

[39] J. J. Halliwell, Phys. Rev. D38 2468 (1988).

[40] M. Henneaux, C. Teitelboim, and J. Vergara, Nuc. Phys. B387 391 (1992).

[41] J. J. Halliwell and J. B. Hartle, Phys. Rev. D43 1170 (1991).

[42] D. L. Wiltshire, in Cosmology: the Physics of the Universe, edited by B. Robson, N. Visvanathan, and W. S. Woolcock (World Scientific, Singapore, 1997).

[43] J. B. Hartle and D. N. Marolf, , Phys. Rev. D56 6247 (1997).

[44] J. J. Halliwell and J. Thorwart, Phys. Rev. D65 104009 (2002).

[45] J. J. Halliwell, unpublished.

[46] H. Embacher, Hadronic J. 21337 (1998).

[47] C. W. Misner, in Relativity, edited by M. Carmeli, S. Fickler, and L. Witten (Plenum, New York, 1970); and in Magic without Magic, edited by J. Klauder (Freeman, San Francisco, 1972).

[48] K. Kuchař, J. Math. Phys. 222640 (1981).

[49] C. Kiefer and H. D. Zeh, Phys. Rev. D51 4145 (1995).

[50] Appendix 3 to chapter 7 of S. Coleman, Aspects of Symmetry (Cambridge University Press, Cambridge, 1984); Section A39.3 of J. Zinn-Justin, Quantum Field Theory and Critical Phenomena, Fourth Edition (Oxford University Press, Oxford, 2002).

[51] J. J. Halliwell, Phys. Rev. D39 2912 (1989). 
[52] T. Padmanabhan, Phys. Rev. D39 2924 (1989).

[53] S. Habib and R. Laflamme, Phys. Rev. D42 4056 (1990).

[54] D. Craig, unpublished.

[55] L. S. Schulman, Techniques and Applications of Path Integration (Wiley, New York, 1981).

[56] H. Kleinert, Path Integrals in Quantum Mechanics, Statistics, and Polymer Physics, Second Edition (World Scientific, Singapore, 1995).

[57] V. P. Maslov and M. V. Fedoriuk, Semi-Classical Approximation in Quantum Mechanics (Reidel, Boston, 1981); R. G. Littlejohn, J. Stat. Phys. 687 (1992); M. Brack and R. K. Bhaduri, Semiclassical Physics (Addison-Wesley, Reading, 1997).

[58] D. Craig and A. Yue, unpublished.

[59] A. Vilenkin, Phys. Rev. D37 888 (1988).

[60] R. P. Feynman, Rev. Mod. Phys. 20267 (1948).

[61] J. B. Hartle and K. Kuchar, Phys. Rev. D34 2323 (1986). 